NASA TECHNICAL MEMORANDUM

(NASA-TH-73890) GAS PATH SEALING IN TURBINE
ENGINES (NASA) 44 P HC AO3/MF A01 CSCL 21E

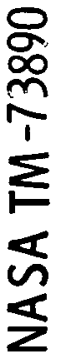

NASA TM-73890

N78-21109

Unclas

G $3 / 07 \quad 12429$

\title{
GAS PATH SEALING IN TURBINE ENGINES
}

by Lavence $P$. Ludwig

Lewts Résearch Center

Cleveland, Ohio 44135

TECHNICAL PAPER to be presented at the AGARD Power, Energetics, and Propulsion Panel Meeting on Seal Technology in Gas Turbine Engines London, England, April 6-7, 1978

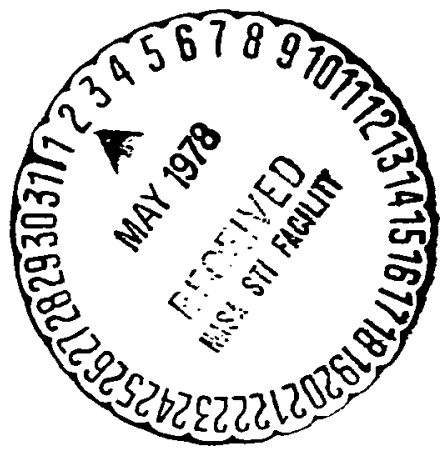

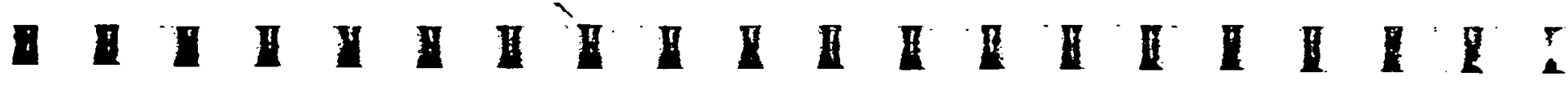


GAS PATH SEALING IN TURBINE ENGINES

\author{
by \\ Lawrence P. Ludwig \\ National Aeronautics and Space Administration \\ Lewis Research Center \\ Cleveland, Ohio 44135
}

\title{
SUMMARY
}

A survey of gas path seals is presented with particular attention given to sealing clearance effects on engine component efficiency. The effects on compressor pressure ratio and stall margin are pointed out. Various caserotor relative displacements, which affect gas path seal clearances, are identified. Forces produced by nonuniform sealing clearances and their effect on rotor stability are discussed qualitatively, and recent work on turbine-bladetip sealing for high temperatures is described. The need for active clearance control and for engine structural analysis is discussed. The functions of the internal-flow system and its seals are reviewed.

\section{INTRODUCTION}

Aircraft gas turbine engines have many sealing locations; along the shaft, over rotor blade tips, and between stages. A large engine may have over 50 (Fig. 1), and the cumulative effect of leakage on engine power, thrust, and aircraft range can be significant. Reference 1 characterizes gas path sealing as a fundamental and continuing problem, which worsens as gas turbine engines advance to higher cycle pressures and temperatures. Of particular interest is the effect of sealing on engine efficiency, which has taken on added interest because of the pending petroleum scarcity and attendant higher prices.

The airline industry in the United States consumes only $10 \%$ of the total petroleum demand in the United States. By 1985 consumption may reach 15\% (Fig. 2 from Ref. 2), but fuel cost may markedly impact the aircraft industry.

Reference 3 emphasizes the need for low fuel consumption now and in the near future because of fuel prices, with fuel reaching $53.5 \%$ of the direct operating cost $(\mathrm{DOC})$ when the price is 50 cents per gallon (see Table I). In the long term, when demand for oil exceeds productive capacity (Ref. 4), a sharp price increase is anticipated by some (see Fig. 3). In general, anticipated fuel scarcity and prices mean that new engines, in addition to the four basic requirements of reliability, durability, low maintenance, and increased power, must also be fuel efficient.

Reference 5 gives additional information on the relative impact of various parameters on DOC and this is shown in Fig. 4, which reveals that DOC is much more sensitive to changes in specific fuel consumption (SFC) than engine weight. Increased fuel scarcity and prices also focus attention on engine performance deterioration, which for high pressure ratio engines is due mainly to the opening up of sealing clearances by erosion and wear.

Engines of the 1950 design era operate at relatively low pressure ratios, and clearances over the blade tips were large enough to avoid rubbing. Modern engines have pressure ratios in the range of $25: 1$, and to preserve efficiency the sealing clearances were reduced. These smaller clearances made rubbing contact at sealing locations inevitable and brought about the introduction of "abradable" materials, which are designed to wear sacrificially.

Abradable materials, which can solve only part of the sealing clearance problem, have not been fully successful because of the erosion and wear of blades. Evidence of this is in airline reports, which note on engine overhaul, that these modern engines deteriorate faster than earlier ones. Typically, high bypass ratio engines have a specific fuel consumption (SFC) increase of 1 to $1 \frac{1}{2} \%$ per year (Ref. 6); periodic overhauls do not fully recover this efficiency loss (Fig. 5). The final result is an engine with a fuel consumption which is 3 to $10 \%$ higher than that of a new engine; Ref. 2 places the average fuel consumption increase at $7 \%$. For a military turbofan Ref. 7 places the SFC increase at near $2 \frac{1}{2} \%$ in 250 hours and indicates that much of this is due to increases in seal leakage. In addition to the effect on efficiency, the sealing clearances also have a significant effect on the compressor stall margin and are directly responsible for "thrust droop" which can be as high as $12 \%$ (Ref. 7 ).

The objective of this study was to survey the present state-of-the-art of turbine engine sealing with attention given to (1) the sensitivity of component efficiency to sealing; (2) the mechanical and thermal effects which control the sealing clearances; and (3) technology needs.

STAR Category 07 
2. SEALING LOCATIONS AND SEAL TYPES

Primary Gas Path, Compressor and Turbine

In general, primary gas path seals perform two functions:

(1) To minimize gas recirculation

(2) To minimize gas leakage out of the primary gas path.

Sealing to minimize gas recirculation is found at the following compressor (Fig. 6) and turbine (Fig. 7 ) locations:

(1) Rotor stages

(a) For unshrouded rotors between blade tips and the case inner wall (Figs. 6(a) and 7(a)) and for shrouded rotors between blade shroud and the case (Figs. $6(\mathrm{~b})$ and 7 (b))

(b) Blade roots and platforms.

(2) Stator stages - For cantilevered vanes between compressor vane tips and rotor (Fig. $6(\mathrm{~b})$ ), and for shrouded vanes between the inner shroud and the rotor labyrinth teeth (Figs. 6(a) and 7 (b)).

(3) Cavities - Rim sealing (Fig. 7 (b)) to prevent hot gas ingestion into the turbine cavities.

Sealing to limit gas loss from the primary gas path occurs at the following locations:

(1) Flanges

(2) Vane pivots (Fig. 6(c)) - Compressor leakage at the vane pivots can be as high as $0.2 \%$ of engine airflow per stage (Ref. 8).

(3) Compressor end seal (Fig. 6(d) from Ref. 9) - Controls cooling and purging flows and rotor thrust balance. Leakage is in the range of $0.6 \%$ of engine airflow in some engines (Ref. 8). Usually most of this leakage is returned to the primary flow in a lower pressure region.

Internal-Flow System

Pressurized air, which is bled off the primary flow, enters the internal-flow system and is used to purge cavities (prevent hot gas ingestion), pressurize bearing compartments, act as a sweep gas to prevent lubricant leakage, cool parts, and balance the rotor thrust. The internal-flow is largely controlled by labyrinth seals; although rubbing carbon seals are sometimes used at the mainshaft sealing locations. Problems arise because wear to these internal-flow system seals changes the amount of air delivered.

Reference 10 points out that increases in pressure ratios, bypass ratios, and turbine temperatures tend to increase the thermodynamic loss chargeable to the internal-flow system; this trend is indicated in Fig. 8 , in which the SFC penalty for an increase internal-flow is plotted as a function of bypass ratio. According to these data the SFC penalty increases when either bypass ratio or pressure ratio is increased. In addition, with the continuing trend to high temperatures there is an associated requirement for more cooling and purging flow.

The marked effect of internal-flow system is also indicated by the study of Ref. 11 on the leakage effects in a small engine having a compressor flow of $2.3 \mathrm{~kg} / \mathrm{sec}(5 \mathrm{lbm} / \mathrm{sec})$. The performance of this engine was calculated for zero clearance and for typical seal clearances assigned to each position. The results (Fig. 9) show that the most significant losses are in the labyrinth seals for which the calculated penalty is a $17 \%$ loss in power and a $7 \frac{1}{2} \%$ increase in SFC.

As mentioned previously, the internal flows are largely controlled by labyrinth seals at various locations such as at the compressor discharge (Fig. 6(d)), high pressure turbine (Fig. 10), and bearing sumps. Labyrinth seals are attractive because of their mechanical simplicity, reliability, and freedom from limitations on axial displacements. Common configurations are the straight and stepped designs (Fig. 11). Both are reasonably effective when clearances are small. But both lose performance fast with wear. When large clearances are dictated by transient thermal conditions, the performance tends to be poor. But the stepped seals are less sensitive to clearance changes, and, in general, have significantly less leakage than the straight design.

To obtain close clearances, the labyrinth seals must be designed to tolerate rubs, and the general practice in aircraft gas turbines is to place labyrinth teeth on the rotor and ideally have all of the wear, due to interference, take place on the stator rub surface. The reasons for attempting to have all of the wear occur in the stator rub surface are: 
(1) In locations in which the rubs tend to be local (accommodation of eccentricity, out-of-roundness, etc.), wear to the stator is a lesser increase in leakage area than the $360^{\circ}$ of area increase which occurs with rotor wear.

(2) In some locations the realtive displacement of the stator and rotor includes axial and radial components that have a correspondence with an engine operating condition. As illustrated in Fig. 12(a), it is possible to accommodate interferences produced by these displacements and yet maintain an acceptable sealing clearance. On the other hand, If the rotor labyrinth teeth wear instead of the stator, the final leakage clearance is increased by the interference depth of the rub.

(3) Stator wear introduces the possibility of designing to obtain stator/rotor interlocking operation in some applications (Fig, $12($ b)).

Labyrinth teeth are generally thin in order to (a) promote sharp edge orifice flow characteristics, (b) limit the amount of heat generated, and (c) restrict the amount of heat conducted into the rotor. Limiting the heat conduction into the rotor precludes a rotor thermal response, which could aggravate the rubbing and lead to a catastrophic fallure.

An important part of the internal-flow system is the engine mainshaft seals, which protect the bearing sumps as illustrated in Fig. 13. Figure 14 shows several shaft seal schematics for the turbine bearing sump location. Here the basic problem is the protection of the bearing sump from the turbine cooling gas. In early engines the cooling gas pressure and temperature was relatively low and a single labyrinth seal, which restricted turbine cooling gas leakege into the sump, was adequate. Single labyrinth seals have been used to pressure differentials of $34 \mathrm{~N} / \mathrm{cm}^{2}$ (50 psi). At these pressures the efficiency loss due to seal leakage was generally insignificant. However, a disadvantage of the labyrinth seal, as compared with the close-clearance seals (circumferential and face), is easier passage of airborne water and dirt into the sump. In addition, for labyrinth seals, reverse pressure drops must be avoided to preclude high oil loss. In fact, a slight positive pressure differential should be maintained to prevent oil leakage.

When high-pressure air is required for turbine cooling, the multiple-labyrinth system (Fig. 14(b)) is often used (in large engines). In this system low-pressure compressor bleed surrounds the sump, and hence, provides thermal protection; leakage into the sump provides the required sump pressurization. The multiple-labyrinth seal system has higher operating temperature and speed capability than rubbing contact seals and has been used to $280 \mathrm{~N} / \mathrm{cm}^{2}$ $(400 \mathrm{psi})$ and $922 \mathrm{~K}\left(1200^{\circ} \mathrm{F}\right)$. However, as engine size decreases, the multiple labyrinths becomes difficult to apply because of space restrictions (vents, bleeds, lines, etc.). Small engines therefore, require other solutions.

Conventional face seal technology (Fig. 14(c)) can be used to replace the multiple labyrinths up to pressures of $90 \mathrm{~N} / \mathrm{cm}^{2}$ (130 psi) and to sliding speeds of $122 \mathrm{~m} / \mathrm{sec}(400 \mathrm{ft} / \mathrm{sec})$. In another arrangement, which is used in some modern large engines, the labyrinth seal next to the bearing is replaced by a face seal (see Figs. 10 and 14(d)); this buffered face seal system has the advantage of relatively low leakage into the bearing sump. Also, bearing sum reverse pressure differentials are more readily tolerated. Further, according to the analysis of Ref. 12, the buffered face seal can significantly lower the efficiency penalty below that of the multilabyrinth seal system. This analysis, which was made for a large transport engine with a 25:1 pressure ratio, showed a leakage loss of $0.88 \%$ of engine airflow for the multilabyrinth seal system. In contrast, the buffered-face seal system bleeds less air from the compressor, and the seal system pressures permit recovery of the bleed loss in the low-pressure turbine. The net result of the analysis was a predicted $0.6 \%$ SFC gain.

In addition to face and labyrinth seals, ring seals are also used for mainshaft sealing. The simplest is the "floating" ring (Fig. 15(a)) which is so named because it is not restrained from moving in a radial direction and, therefore, can operate with less leakage gap clearance than the conventional labyrinth seals (Ref. 13).

Rubbing, circumferential seals operate with very low gas leakages and are therefore attractive, but the pressure differential capability is generally low because of the rubbing contact. The simplest circumferential rubbing seal is composed of a segmented carbon ring (three $120^{\circ}$ segments) held together by a garter spring on the outside diameter ( $\mathrm{Fig}$. 15(b)). The gaps between the adjacent ends of the segments are a source of air leakage into the bearing cavity.

Other circumferential seal designs incorporate multiple rings with overlapping joints to eliminate the leakage at the gaps between the segments. Pressure balancing of the segments maximizes the pressure and speed capability (Fig. 15(c)). Reference 14 reports successful operation of a pressure balanced circumferential seal to $58 \mathrm{~N} / \mathrm{cm}^{2}$ (85 psi) and $73 \mathrm{~m} / \mathrm{sec}(240 \mathrm{ft} / \mathrm{sec})$.

In partial summary of this section, mainshaft sealing at the turbine bearing locations is accomplished by several different designs: (a) multilabyrinth seals, (b) rubbing contact seals, or (c) a combined system of a rubbing contact and labyrinth seals. Labyrinth seals have high leakage, which can be a performance penalty, and in small engines with restricted space the high leakage rates are difficult to accommodate. On the other hand, contact seals 
have low leakage but have limited pressure and speed capabilities. A need, therefore, exists for mainshaft seals which have low leakage yet are able to function at the pressures and speeds expected in advanced engines (pressure ratios in the range of 45:1 (Ref. 15) and seal sliding speed could reach 183 to $244 \mathrm{~m} / \mathrm{sec}(600$ to $800 \mathrm{ft} / \mathrm{sec}$ )).

\section{LEAKAGE FLOWS}

Blade and Vane Tips Leakage Flows

Leakage flow between the blade (or vane) tip and the adjacent wall is due to

(1) The pressure difference between the suction and pressure side of the blade

(2) The relative wall movement.

The velocity gradient of the flow relative to the blade is represented by the classical Couette flow profile indicated in Fig. 16. And the velocity profile produced by the pressure difference is probably similar to that of a slit type of orifice; that is, the classical fully developed profile is not obtained because the ratio of blade thickness to clearance is small.

In the case of a compressor blade or vane tip, the pressure difference and the relative wall movement both act to cause leakage in the same direction; in a turbine these two effects oppose each other. In general, the leakage over the tips flows in a direction tending toward a normal to the main flow, and the resulting interaction of these two flows is described by some (Refs. 16 and 17) as a tip vortex such as that depicted in Fig. 17. Reference 17 gives a detailed description of the tip leakage interaction with the boundary layer. Figure 18 shows that tip leakage flow of relatively high energy tends to turn back the lower energy secondary-flow and form a core of low energy fluid; this core is pushed further toward the blade pressure side as the tip leakage increases. Observations made by the author of Ref. 18 using a multistage axial flow hydraulic pump confirm that this slot discharge rolls up into a vortex. Losses are considered to arise because the velocity component normal to the chord is not recovered.

Reference 19 presents a method for predicting tip clearance effects in turbomachinery, and a semiempirical expression is formulated using a model in which the lift is uniform along the span and only a part of the bound vortex is shed along the tip. Boundary conditions are satisfied through use of image vortices, and the associated flow is used to predict induced drag. The semiempirical expression for efficiency drop $\Delta \eta$ from the analysis by Ref. 19 is

$$
\Delta \eta=\frac{0.7 \lambda \psi}{\cos \beta_{\mathrm{m}}}\left(1+10 \sqrt{\frac{\varphi \lambda \mathrm{A}}{\psi \cos \beta_{\mathrm{m}}}}\right)
$$

in which

$$
\begin{gathered}
\lambda=\mathrm{c} / \mathrm{s}, \text { tip clearance/blade height ratio } \\
\psi=\text { blade loading }=\frac{2 c_{\mathrm{p}} \Delta \mathrm{T}_{\mathrm{o}}}{\rho \mathrm{U}^{2}}=\frac{2\left(\Delta \mathrm{P}_{\mathrm{o}}\right)_{\text {isen }}}{\rho \mathrm{U}^{2}} \\
\beta_{\mathrm{m}}=\text { mean air angle } \\
\mathrm{A}=\text { aspect ratio of blade } \\
\varphi=\text { flow coefficient }=\frac{\mathrm{V}_{2}}{\mathrm{i}}=\frac{\text { fluid exit velocity }}{\text { blade speed }}
\end{gathered}
$$

The preceding formula is for a cascade and does not include rotation effects. Also, the formula's applicability needs to be established over a wide range of design parameters. This suggests a need for a better mathematical model which would accurately reflect the effects of the various parameters. The complexity of the flow and the interaction of the many parameters (such as chord length, blade loading, aspect ratio, Mach number, rotational speed, etc.) suggests that the expression will continue to be semiempirical.

\section{Labyrinth Seal Flows}

Two basic approaches are used to predict labyrinth seal leakages; one is based on a pipe friction model, and the other, which seems to have wider acceptance, is based on a series of throttlings. The physics of the flow is illustrated in Figs. 19 and 20. Ideally, the kinetic energy increase across each annular orifice (stations 1 to 2 in Fig. 19) is completely dissipated in the cavity (stations 2 to 3 ). In actuality, the kinetic energy dissipation is not 
complete, and Egli (Ref. 20) introduced the concept of "carry-over" (see fluid dynamic model in Fig. 20) in order to account for the incomplete dissipation of kinetic energy in straight labyrinth seals. Reference 21 gives the following convenient form of an Egli equation for calculating leakage, $\mathrm{W}$ :

$$
\mathrm{W}=\mathrm{A} \varphi \alpha \gamma \sqrt{\mathrm{g} \rho_{\mathrm{u}} \mathrm{P}_{\mathrm{u}}}
$$

where $A$ is the leakage area; $\varphi$ is the flow function and a function of $N, P_{d} / P_{u^{*}} \alpha$ is the discharge coefficient and a function of $t, c / t ; \gamma$ is the carry-over factor and a function of $N, c / s ; P_{u}$ is the upstream pressure; $\rho_{u}$ is the upstream density; $N$ is the number of labyrinth teeth; $c$ is the clearance; $t$ is the tooth thickness; $G$ is the tooth spacing; and $P_{d}$ is the downstream pressure. The values of $\varphi, \alpha$, and $\gamma$ are obtained from the graphs in Fig. 21 from Ref. 22.

From the flow function curve (Fig. 21) it is seen that increasing the number of throttling decreases flow but that the gain beyond six teeth is small. This is one reason why labyrinth seals in aircraft engines generally have six or fewer teeth.

The discharge coefficient $\alpha$ is affected by tooth shape, tip thickness to clearance ratio, pressure ratio across the tooth, and eccentricity. Extensive experiments (Ref. 1) have shown more complexity than indicated in Fig. 21(b). For example, the effects of tooth shape on discharge coefficient is given in Fig. 22; these data show a significant sensitivity to tooth shape. Sharp corners provide the lowest discharge coefficient; however, from a practical viewpoint, labyrinth seals generally rub, and rounded corners are common, particularly if the stator rub material is not easily abraded. Thus the discharge coefficient may approach that of a nozzle. (Please read Ref. 1 for a more complete coverage of discharge coefficient.)

Experimental data are often expressed in terms of an overall flow parameter $\psi$, which includes the flow function $\varphi$, discharge coefficient $\alpha$, and the carry-over factor $\gamma$. Therefore, equation (2) can be expressed as

$$
\mathrm{W}=\psi \mathrm{A} \frac{\mathrm{P}_{\mathrm{u}}}{\sqrt{\mathrm{T}_{\mathrm{u}}}}
$$

An example of experimental data for a labyrinth seal with four teeth is shown in Fig. 23, in which the flow parameter $\psi$ is plotted as a function of pressure ratio. These data indicate choking above a pressure ratio of about 2.5. Most engine manufacturers have considerable labyrinth seal experimental data, and their semiempirical analytical techniques based on these data (e.g., Figs. 22 and 23) permit accurate prediction of the leakage. The major error probably comes in clearance estimation.

The potential payoff of reduced leakage validates the continuing search for improved labyrinth seals. Potential reductions may result from the following:

(1) Stator rub materials that increase dissipation (data in Ref, 23 show that a honeycomb stator rub surface is most promising)

(2) Tooth edges that retain corner sharpness under rubbing and erosion conditions

(3) Geometries that reduce carry-over effects

(4) Theoretical studies for added insight with less dependence on empirical data (provide optimum designs)

(5) Closer running clearances.

\section{SEALING CLEARANCE SENSITIVITY}

Compressor System Sealing

Many parameters are associated with blade tip clearance leakage, some are blade loading, tip thickness, aspect ratio, wall relative speed, and Mach number. But the one favored as the most significant by many investigators is the ratio of clearance $c$ to blade height $s$. References 1,24 , and 25 contain experimental data on relatively large diameter fan and compressor efficiency as a function of either clearance or c/s ratio. The data of Ref. 1 are in terms of a penalty on a baseline efficiency at near zero clearance ( $F$ ig. 24); the absolute levels of efficiency are not given. On the other hand, data in Refs. 24 and 25 are in terms of efficiency levels, and the penalty on an efficiency level at near zero clearance can be obtained through extrapolation. This, however, requires some knowledge of the behavior of the efficiency curve at small $\mathrm{c} / \mathrm{s}$ ratios. 
Inspection of the relationship between efficiency penalty and $c / s$ ratio in Fig. 24 reveals these general features:

(1) The initial slope of the curve at very low $\mathrm{c} / \mathrm{s}$ ratios $(\approx 0.0005)$ has about a $10 \%$ efficiency penalty for each 0.01 -increment in $\mathrm{c} / \mathrm{s}$ ratio.

(2) The final slope of the curve is constant beyond a $\mathrm{c} / \mathrm{s}$ ratio of about 0.008 and has a magnitude of about $2 \%$ efficiency penalty for each 0.01 -increment in $\mathrm{c} / \mathrm{s}$ ratio.

(3) There is no indication of an optimum finite clearance from an efficiency standpoint (although Ref. 19 presents a heuristic argument for the existance of an optimum clearance).

Reference 25 shows experimental multistage compressor efficiency as a function of five different clearances (see Fig. 25). The closest clearance provided a c/s ratio of 0.011 . This compressor, referred to in Ref. 25 as "Alice," had eight stages and ran at a mean blade speed of $93.9 \mathrm{~m} / \mathrm{sec}(308 \mathrm{ft} / \mathrm{sec})$. With reference to Fig. 25 the slope of the curve is constant over a wide range of $\mathrm{c} / \mathrm{s}$ and has a magnitude of 1.95. This is added evidence that the final slope of the sensitivity curve shown in Fig. 24 may have general applicability.

Data on clearance effects of single-stage fans (Ref. 24) ranges between a $\mathrm{c} / \mathrm{s}$ of 0.0014 to 0.0118 . Thus the data, which are shown in Fig. 26, span a region in which the slope of the sensitivity curve is rapidly changing, whereas the data for the "Alice" compressor is entirely within the final constant slope region. When drawing the curve in Fig. 26, it was assumed that the initial and final slopes would be the same as those in Fig. 24. These assumptions seemed to agree with the general trends of the curve and permitted a short extrapolation to obtain a baseline efficiency at a $\mathrm{c} / \mathrm{s}$ of 0.0005 . This extrapolation permits the data to be expressed in terms of efficiency penalty from a baseline. A comparison with that from Ref. 1 is given in Fig. 27. Data from Ref. 24 indicate a greater penalty, and the variances suggest additional studies are needed. In particular, data are needed at small $\mathrm{c} / \mathrm{s} \mathrm{ratios}$.

Generally, the effect of leakage is more significant in the small engines because, the leakage flow area is relatively larger. However, a significant problem in large engines is blade tip rubbing from case bending (Ref. 26) and out-of-roundness. (This is discussed in a later section.) Even though small engines tend to be stiff and hold roundness, small engine compressor efficiency tends to be about $5 \%$ less than that for large engines (Ref. 27). This fact places increased emphasis on reducing penalties caused by clearance effects.

For a small axial compressor with a diameter of $11.770 \mathrm{~cm}(4.634 \mathrm{in}$.$) , Ref. 28$ shows a maximum stage efficiency at design speed of $83.3 \%$; the running tip clearance was $0.78 \%$ of the rotor blade mean span. And when the rotor diameter was machined down to increase the clearance to $2.14 \%$ of blade span, the efficiency decreased by 5.5 points. Additional data on small axial compressors are found in Ref. 27, which contains a compilation of various sources. There are variances between the sets of data, but all data indicate a serious degradation of efficiency due to clearances. A typical rate is a 1.5-percentage-point loss in efficiency for each increase of 0.01 in clearance to span ratio.

Clearance effect data for centrifugal compressors are given by Refs. 27, 29, and 30; typical data are shown in Fig. 28. For axial clearance changes at the tip, data from Ref. 27 show a 1.00-percentage-point loss for each 0.01 increase in $\mathrm{c} / \mathrm{s}$ ratio ( $\mathrm{s}$ is the axial depth of the blade at the tip). In contrast, Refs. 29 and 30 indicate a 0.37 to 0.49 percentage point loss.

As with the larger diameter compressors, the data for small-diameter compressors (axial and radial) show considerable effect on efficiency however the variances suggest that additional studies are needed. Since data for the small compressors are for $\mathrm{c} / \mathrm{s}$ ratios above 0.02 , there remains the question of how efficiency changes with clearance below a $\mathrm{c} / \mathrm{s}$ ratio of 0.02 .

In some engine designs the compressor stator vanes are cantilevered (see Fig. 6(b)), and the clearance effects are somewhat similar to those of the blade tips. Reference 1 gives data on efficiency penalty as functions of clearance to blade height ratio, and these data are shown in Fig. 29. In comparison with the blade tip data of Fig. 27, the penalties are not nearly as severe.

The other type of construction used in stator vane sealing is the inner diameter shroud depicted in Fig. 6(a). Compared with the cantilevered constructions, the inner shroud and associated labyrinth seal form annular passages underneath the primary flow path, and these passages allow circumferential flow; the net result is detrimental effect on stall margin.

Figure 30 (from Ref. 1) shows efficiency penalty for clearances in the stator vane/inner shroud type of construction. The data in Fig. 30 are for labyrinth seals having two knife edges with no carry-over blockage (no steps). A comparison of the penalties in Fig. 30 with those for cantilevered stator vanes shows that the inner shroud construction has less effect on compressor efficiency. 
In regard to the preferred type of vane sealing, the inner shroud construction produces undesirable annular passages which allow circumferential flow; on the other hand, inner shroud construction provides greater structural integrity. This is important from an F.O.D. and aeroelastic stability standpoint. Further, in cantilevered construction, the clearance between the vane tips and rotor must be selected to avoid hard rubs, and therefore is generally larger than for the inner shroud construction. Vane tip rubbing has, in some cases, triggered a rotor thermal response feedback which increased the rubbing severity until catastrophic failure. However, an additional factor which favors the cantilevered construction is the significantly lower boundary-layer temperature (at the inner gas path) than with the shrouded stator vanes (Ref. 10).

In addition to the effect on efficiency, blade tip clearances also affect the stall margin and the pressure ratio. Reference 24 supplies valuable data in this regard, and some are shown in Fig. 31. The steep slope of both curves, as clearances are decreased, suggests a large potential improvement if close running clearances could be obtained. Reference 28 also provides data on stall margin as affected by clearance for a single stage fan with a relatively low tip speed of $68.1 \mathrm{~m} / \mathrm{sec}(223.4 \mathrm{ft} / \mathrm{sec})$. Tip clearances of $0.3,0.6$, and $1.2 \mathrm{~mm}(0.012,0.024$, and $0.047 \mathrm{in}$.) were used, and these gave $\mathrm{c} / \mathrm{s}$ values of $0.0033,0.0067$, and 0.0133 . Using the largest clearance as a basis, the stall margin improved by $4.7 \%$ when the clearance was reduced to $0.6 \mathrm{~mm}(0.024 \mathrm{in}$.) and improved $7.1 \%$ for the $0.3 \mathrm{~mm}$ (0.012 in.) clearance.

Turbine System Sealing

The decrease in turbine efficiency associated with an increase in clearance is due, in part, to unloading of the blade by leakage over the tip, and to the increase in flow area over the blade tips. In addition, when the increase in clearance occurs because of blade wear, an added loss is associated with the decrease in active blade area. In this regard, it is preferable to have the case wear instead of the blade. This is also true of compressors. These two idealized extremes, wear to the blade with none to the case, and vice versa, are illustrated in Fig. 32. Interference between the blades and case, which leads to wear, is usually caused by transient thermal differences between the rotor and the case. This is discussed in a later section.

Data from Ref. 31, which is repeated in Fig. 33, reveals that changes in clearances are accompanied by significant changes in both exit flow angle and local efficiencies over the entire blade height. These data are for different clearances (up to $8 \%$ of flow passage height), which were obtained by machining down the blade tips. Near the tip the exit flow angles showed a large change, nearly $40^{\circ}$. In addition, the average flow angle across the flow passage changes. This indicates that underturning accompanies increases in leakage through the clearances. Also, Fig. 33 (b) reveals a large variation in local efficiency across the entire blade height with the greatest change occurring between the mean radius and the tip. Thus, clearance can affect the flow across the entire blade span.

References 32 to 34 contain experimental data on the effect of tip clearances on turbine efficiency, and Ref. 34 contains a comparison of these data, which is shown in Fig. 34. These comparisons, which are expressed as fractions of zero-tip-clearance efficiency, were obtained by linear extrapolation to zero clearance. Inspection of Fig. 34 reveals, in general, a significant impact of tip clearance on turbine efficiency, and the data show that a greater loss is associated with reaction turbines, than with impulse types, apparently because of the higher pressure difference across the blade. In this regard, the reaction turbine used in studies of Ref. 34 had an efficiency loss of $2.0 \%$ for a clearance change of $1 \%$ of blade height when the clearance change was produced by machining down the blade tips. But when the clearance was obtained by machining out the case, and with the blade tip diameter equal to the casing inside diameter (see Fig. 35(a)), the efficiency slope for a reaction turbine was $1.5 \%$ for a clearance change of $1 \%$ of blade height.

For these machined case configurations the optimum efficiency occurred when the blade tip diameter was equal to the case inside diameter (Fig. 35(a)); when the blade tip was below the case inside diameter or when the blade protruded into the machined recess (Fig. 35(b)), the losses were greater. For example, the data of Ref. 34 show that for a clearance of $2 \%$ of the blade height, the efficiency drops from $97 \%$ for zero blade extension into the recess (Fig. $35(\mathrm{a})$ ) to $96 \%$ for a blade extension into the recess of $3.5 \%$ of blade height (Fig. $35(\mathrm{~b})$ ).

Radial-inflow turbine clearance data are reported in Ref. 35. Studies included the effect of axial clearances at the entrance and exit and the effect of radial clearances. The data reveal sensitivity to radial clearance comparable with that for the axial flow turbines.

Influence Coefficients

The real measure of the effect of seal leakages is in its effect on specific fuel consumption (SFC) which is inversely proportional to the product of cycle and propulsive efficiencies (Ref. 15). The cycle efficiency is limited mainly by gas generator component efficiency and by airbleed requirements for hot-section cooling. For a high bypass engine the propulsive efficiency depends, to a great extent, on the efficiencies of the components acting on the 
bypass stream, thus the fan and low-pressure turbine efficiencies are a major influence.

Reference 15 provides data on the influence of various high bypass engine component efficiencies on the specific fuel consumption and these data are repeated in Fig. 36. It is noteworthy that for a high bypass engine a low-pressure turbine (LPT) efficiency change causes the greatest change in SFC; a $0.86 \%$ improvement in SFC for each percentage point increase in turbine efficiency. The high pressure compressor (HPC) and turbine (HPT) each provide about $0.5 \%$ SFC change for each $1 \%$ change in component efficiency.

Reference 6 provides influence coefficient type data, in terms of fuel consumption sensitivity to component deterioration, for the JT3D-3B and JT8D-9 engines. These data were determined by mathematical models of engine performance and show, for example, the following fuel flow increases for a $1 \%$ deterioration in each of the following component efficiencies:

$\begin{array}{cc}\text { Component } & \text { Fuel flow increase, } \\ & \% \\ \text { Fan } & 0.29 \\ \text { HPC } & .51 \\ \text { HPT } & .58 \\ \text { LPT } & .69\end{array}$

Interestingly, a $1 \%$ increase in HPT flange leakage resulted in a predicted increase in fuel flow of $1.22 \%$. This points up not only the significance of flange sealing but also the large influence of any high pressure air loss from the cycle. In this regard, vane pivot leakage and HPC flange leakage have a similar influence. Also, it suggests that variable vanes for HPT of advanced engines be given close design study in regard to sealing effectiveness.

\section{SEALING CLEARANCES}

In previous sections the need for close clearances in modern gas turbine engines was stressed. A basic problem is that the radial displacements of the case and rotor are, in general, much greater than the desired operating clearances. An approximate ordering of the more significant transient and nontransient displacements which affect clearance are

(1) Thermal response of the case and rotor

(2) Centrifugal and gyroscopic loads

(3) Surge/stall displacements

(4) Thrust, aerodynamic, maneuver, gust, and landing loads

(5) Ovalization and out-of-roundness due to nonaxisymmetric structures, loads, and temperatures

(6) Case/rotor vibration and shaft thermal bow

(7) Assembly eccentricities

ORIGINAL PAGE IS OF POOR QUALITY

(8) Machining tolerance variations.

Abradable materials in labyrinth seals and over compressor blade tips can make accommodations for allow corrections) for assembly eccentricities, machining tolerance variation between assemblies, and out-of-roundness. However, the other effects listed must be controlled through the integrated mechanical design of the whole rotor-case.

The transient thermal response of the case and the rotor is of major concern, and this response, along with the centrifugal loading, is one of the most important factors in setting the final cruise clearance. In general, the thermal response of the case and rotor are not the same because of differences in mass, cooling-air circulation, heat transfer, and material. Figure 37 illustrates the general problem of relative case/rotor displacements as it is affected by thermal response and centrifugal loading. The case tends to have a much faster thermal response to the gas path stream temperature than the rotor. The rotor growth is initially due to centrifugal force during acceleration, and if assembly clearances are too small, a rub will occur in the early part of the acceleration. On deceleration, the case's relatively fast thermal response will cause rubs if full power is demanded after a period of low power (such as in an aborted landing). This is also illustrated in Fig. 37 in which the relatively fast case response has reduced the clearance to a magnitude less than the rotor displacement due to centrifugal force. The problem can be mitigated by closer thermal matching of the case and rotor, but not completely solved. If very close cruise clearances are going to be obtained then some type of active clearance control is needed to eliminate the rub potential. In this regard, Ref. 36 describes a case cooling system, now being used in current commercial engines, which reduces the cruise clearances in the high pressure turbine. This cooling system is automatically shut off to 
increase the clearance during potential rub situations.

As mentioned previously, a problem in some engines is a loss in thrust on engine acceleration. This is thought to be due to the opening up of certain critical sealing clearances and has been attributed to the fast sealing case thermal response. Magnitudes of thrust loss in the range of $12 \%$ have been reported (Ref. 7 ) for conditions in which throttle advance is made on a "cold" engine; for example, when the engine is started, taxied to the end of the runway, and then accelerated for takeoff.

Typical transient clearances for a high bypass engine are given in Ref. 37 for a first-stage high-pressure turbine; these data (shown in Fig. 38) show blade tip clearance changes of about $0.76 \mathrm{~mm}(0.030 \mathrm{in}$.) over a period of 400 seconds.

As mentioned previously, large engines have relatively flexible cases and rotors; these are inherent in largediameter, flight weight structures. As a result, various thrust, aerodynamic, and gyroscopic loads cause appreciable relative displacements at certain sealing locations. An example pointed out in Ref. 26 is the engine case bending (large high bypass engine) resulting from aerodynamic loads on the inlet cowl (see illustration in Fig. 39) and from the thrust loading. These inlet lift and thrust loads, which are large during climb-out after takeoff, cause rubbing at the $12 \mathrm{o}^{\prime}$ clock position in the high-pressure compressor and at the $6 \mathrm{o}^{\prime}$ clock position in the fan.

Reference 26 also points out that vibration characteristics (response to gust, takeoff, turbulence and maneuver loads) are of fundamental importance in propulsion system design and that a piecemeal, component-by-component analysis is not sufficient because of coupling effects. The analytical method described in Ref. 26 covers the engine and airframe related components and has these two main procedural steps:

(1) The use of static models of installed propulsion system and steady-state loads to calculate preliminary magnitudes of stresses and deflections

(2) The refinement of the analysis through the use of a dynamic model of an installed propulsion system with transient flight loads, gyroscopic forces, and rotor unbalance to calculate stresses, deflections, and frequencies.

The analytical model (Ref. 38) includes the probability of exceeding a flight load, which will cause a blade tip rub; this is expressed in terms of "exceedances" (rubs) per 1000 flights. The data in Ref. 38 show that exceedances are a function of engine position, and show most of the change in clearance due to rubs occurring during the first 10 flights. This agrees with a conclusion from the study in Ref. 6 that the deterioration consisted of two portions, an initial rapid rate (wear in), which opens up the clearances, and then a slower rate, which is dependent on erosion rate and the number of adverse events (compressor stalls, hard landings, severe gusts, etc.).

In small and large engines a major consideration is the roundness of the case. For example, there could be transient effects such as the response of a horizontally split compressor case to a thermal change. In general, horizontal flanges at the split line have a different thermal response from the rest of the case, and the flange also causes the case stiffness to be nonaxisymmetric. In the hot section out-of-roundness is caused by (1) nonuniform temperatures, (2) nonaxisymmetric structures, (3) localized flange leakage, (4) nonuniform distribution of cooling air, and (5) localized flow path gaps (hot-gas recirculation). Engine operation data indicate that hot section out-ofroundness may be in the range of $0.001 \mathrm{~cm}(0.0004 \mathrm{in}$.) per centimeter of diameter. Thus, the out-of-roundness may be greater than the desired operating tip gap. And as turbine temperatures and pressures increase, both the out-of-roundness tendency and performance penalty will increase. A partial solution to out-of-roundness, of course, is in obtaining more axisymmetric temperature distribution. Also, it should be noted that out-of-roundness can be caused by strains in adjacent cases; that is, a maldistribution of temperature in one case section can influence the roundness of adjacent case sections.

Clearances can be affected by rotor and stator vibrations. Shaft dynamics motions due to rigid rotor and flexible rotor unbalance (see Table Ii) have received considerable attention and are amenable to analysis (Refs. 39 and 40 ). Modern analytical methods and shaft balancing techniques generally insure a trouble free design from a rigid rotor critical speed standpoint. Large engines generally operate above the rigid rotor critical and below the flexible rotor mode, and typical radial displacements at operating speed due to rotor unbalance are reported to be in the range of $0.010 \mathrm{~cm}(0.004 \mathrm{in}$.) in large engines. Thus in a well designed system, radial displacement due to critical speed is usually not a major factor in sealing clearances. Some small engines run above a flexible rotor mode, and recent advances in multiplane balancing techniques (Ref. 41) promise small operational displacements. However, there remain questions on response of flexible rotor under adverse conditions such as surge/stall or blade out operation.

There is concern that nonsynchronous whirl (see Table II) could be the source of clearance changes, especially in high-pressure systems. Figure 40 illustrates the general principle for a labyrinth seal. The pressure field within the labyrinth seal will, in general, not be symmetric about a plane (section A-A in Fig. 40) passing through the direction of eccentricity because of preswirl and the nonuniform clearances. (A complicating factor in labyrinth 
seal is axial clearance variation due to convergence, divergence, or axial misalignment.) Since the pressure field is not symmetric about section A-A (Fig. 40), a force component will exist which is transverse to the eccentricity direction, which will tend to produce a self-excited whirl.

A similar principle applies to the case of blade whirl forces; because of nonuniform clearances and associated leakages, blade loading is not uniform (lower loading where the tip gap is larger) and the net result is an aerodynamic force transverse to the eccentricity direction.

An additional potential source of vibration is rubbing friction induced whirl. This is a complex case/rotor interaction which has not been well investigated.

The necessary use of thin sections and light-weight construction has introduced potential vibratory motions of the labyrinth seal structure, especially in larger sizes. Although fatigue cracking of the labyrinth structure is usually the final result, clearance changes with associated wear can also be produced. Instabilities are reported to have occurred when the wave speed of vibration of the rotating labyrinth component was in resonance with the flexural wave speed of the stator shroud. To preclude failure due to resonance with a flexural mode in the stator, Ref. 9 recommends stators that have a minimum angular velocity for all flexural modes at least $25 \%$ higher than the rotor speed at $100 \%$ operation. This may require experimental verification of the predicted frequencies since some stator structures are quite complex.

In addition Ref. 9 makes the interesting observation that labyrinth stator and rotor components do not have fatigue failures when supported on the discharge end. (See Fig. 41.)

Clearance management in the various seals presents a difficult engineering problem, which involves the entire rotor/case assembly, airframe integration, and operational flight loads. In modern engines knowledge of the operating clearances is inferred from rub wear patterns and engine operating history. Direct clearance measurement comes from various types of probes (capacitance, mechanical touch, laser) and from high energy X-radiography, which is capable of measuring sealing clearances within an accuracy of $\pm 0.07 \mathrm{~mm}( \pm 0.003 \mathrm{in}$.) (Ref. 42). In this regard Ref. 42 claims both steady-state and transient clearance can be measured and useful data obtained on

(1) Axial and radial clearances of labyrinth seals

(2) Radial blade tip clearances

(3) Rotor/stator axial clearances

(4) Component deflections.

Reference 43 describes a unique laser optical probe, which is capable of measuring blade tip clearances in compressors and turbines. (For turbine applications a small cooling flow of nitrogen gas is used to keep probe internal parts cool.) The principal of operation is based on reflected light triangulation as indicated in Fig. 42 from Ref. 43. Light from a point source is reflected from each blade tip as it passes by the probe, and the clearance reading is an average of all the blades (current research looks at individual blade clearances). A change in clearance causes a shift of the reflected light such that it falls on a different section of the output fiber optic bundle. Accuracies of $\neq 0.025 \mathrm{~mm}(0.001 \mathrm{in.})$ are claimed, and a probe temperature environment of $1311 \mathrm{~K}\left(1900^{\circ} \mathrm{F}\right)$ can be tolerated. A significant feature is that the high response of the system $\left(0.4 \times 10^{-6} \mathrm{sec}\right)$ permits transient clearance measurements.

\section{GAS PATH SEALING MATERIALS}

If gas path sealing clearances are going to be reduced to a practical minimum, some rubbing contact must be tolerated in order to compensate for eccentricities, machining tolerances, out-of-roundness, vibrations, etc. Ideally these rubbing contacts may be classified into the following two types:

(1) Low energy rub - Low-energy rubs obtained several ways. One is through the use of abradable materials, which are designed to wear instead of producing wear in the blade or labyrinth tooth these approaches are discussed later).

(2) Abrasive - Drum rotors generally have bonded abrasive coatings which protect the rotor from wear and promote vane tip wear in case of interference. These coatings, which are usually hard oxides, also mitigate thermally induced expansions of the rotor, which would aggravate the rub and, therefore, induce thermal feedback which could end in a catastrophic failure.

A major factor that controls rub material selection is the operating temperature. In the colder sections (fan and low pressure turbine) sprayed and molded polymer systems are suitable. But in the high-pressure compressor, the higher temperatures require metal systems such as sprayed nickel/graphite. The high-pressure turbine 
requires very high temperature oxidation resistant materials which, in general, are not abradable and do not provide low energy rubs. Finally, the low-pressure turbine can use some of the materials suitable for the high pressure compressor but honeycomb shrouds are the most popular.

Rub material for over fan blade tip should be abradable or have a low-energy rub property to prevent blade wear. In addition, the fan rub material is usually configured to enhance aerodynamic stability (improve fan stall margin) and acoustical damping. One common shroud material used is aluminum honeycomb, which is readily deformed by fan blade penetration. However, wear to blade tips can be a problem; further, the honeycomb material, which is a rough surface from an aerodynamic standpoint, has an associated efficiency penalty as compared with smooth rub material. Reference 44 reports a 2.5 percentage point loss for honeycomb rub material as compared to a fan with a smooth shroud. The stall margin, however, improved $12 \%$ (see Fig. 43).

Other types of fan shroud rub material are elastomer and polymer base composites, which sometimes contain fillers of hollow glass spheres to improve abradability. These rub shrouds usually contain grooves or slots, which improve the stall margin by stabilizing the fan flow near the tip. Considerable data have been published (see Ref 8 . 45 to 47 ) on the effectiveness of various geometric patterns in the fan shroud. A potential problem with use of some polymeric type materials in the low-pressure compression system is the considerable amount of rub material dust created during a very hard rub under an adverse operating condition. This dust has, on occasion, exploded in the high-pressure compressor.

In the high-pressure compressor higher temperature shroud materials (metal, graphite, etc.) are required because the discharge temperatures are near $922 \mathrm{~K}\left(1200^{\circ} \mathrm{F}\right)$ in some modern engines, and will be even higher in advanced engines. Currently used rub materials over blade tips (outer air sealing) are designed to mitigate blade wear by serving as low-energy rub or sacrificial material. Three different types of low-energy rub materials are used (Fig. 44). The sintered metal system (density 30 to $40 \%$ ) of Fig. 44(a) is representative of an abradable type material, since blade (or labyrinth tooth) penetration breaks off sintered particles. The material effectiveness is often measured by the ratio of material wear to blade wear; a ratio of 10:1 being considered a satisfactory abradability property. Abradability can be readily achieved by lowering density, but this increases the susceptibility to erosion. Thus, a basic difficulty is obtaining acceptable abradability yet maintaining adequate erosion resistance.

The sintered metal fibers depicted in Fig. 44(b) are plastically deformable rather than abradable and are usually about $20 \%$ dense. Blade (or labyrinth tooth) penetration may densify the material (as indicated schematically) and this increases the rub intensity.

A third type used in outer air sealing is the low-shear-strength $100 \%$ dense material (Fig. 44(c)); sprayed aluminum is an example. Blade penetration readily machines away the easily sheared material without excessive wear to the blades. However, the machining debris tends to stick on downstream airfoils and cause aerodynamic losses.

Studies of Ref. 48 indicate a tendency for blade wear when the penetration rate is low (typical blade penetration rates range between 0.00025 to $0.025 \mathrm{~cm} / \mathrm{sec}(0.0001$ to $0.01 \mathrm{in} / \mathrm{sec})$. As yet, a fully satisfactory material has not been developed for outer gas path sealing. blade wear, erosion or aerodynamic loss remain consistent problems. None have all the desirable properties, which are:

(1) No blade wear

(2) Low energy rub a general problem

(3) Innocuous debris (a problem in sprayed aluminum and some polymers)

(4) Erosion resistance

(5) Impermeability a problem in porous materials OBIGINAL PAGE IS
OF POOR QUALITY

(6) Smooth surface $\int$

(7) Easy repair (a problem in brazed assemblies)

Laby rinth shrouds for compressor inner gas path sealing are depicted in Fig. 45; all are designed to produco a low energy rub as compared with a $100 \%$ dense metal shroud. In the high speed rubs, which take place between the shrouds and rotor, the rub mechanisms and associated wear are not well characterized for currently used material couples. Theoretical studies (Ref. 49) on rotor/shroud interaction predict the formation of "thermal bumps" or hot spots which apparently govern the wear process. These hot spots are the result of thermal-elastic surface instabilities produced by contact. For example, experimental data show that when a labyrinth knife edge rubs against a shroud segment, the rubbing can take place over just a small segment $\left(\sim 5^{\circ}\right.$ arc) of the $360^{\circ}$ of tooth edge (see Fig. 46). Thus, the heat input is highly localized, and a local thermal bump is generated which expands, 
rubs harder, and finally wears away. This is then followed by rubbing over a second small segment which grows and then wears, etc. Evidence of localized rubbing is indicated by the heat discoloration of the tooth edge. This type of local rub interaction has been investigated from a fundamental standpoint, and a considerable body of data exist, for example, see Refs. 50 and 51 .

Thermal elastic instabilities apparently occur on the blade tips as well as on the casing rub material. The most adverse operating environment from a sealing viewpoint is in the high-pressure turbine. Here the gas temperatures in some modern engines are in the range of $1700 \mathrm{~K}\left(2600^{\circ} \mathrm{F}\right)$. In addition to the losses due to blade tip clearances, which were previously pointed out, there is a considerable efficiency penalty associated with cooling of the turbine case rub shroud material (outer air seal). In small engines this rub shroud is a single ring; in large engines it is constructed of segments. Typically each segment is impingement cooled and is held in place by a cooler outer case structure. Figure 7 (a) shows, in cross-section, a typical segment and associated attachment structure.

Turbine rub shroud materials used are generally not abradable and turbine blade tip wear is a general problem. Transfer of the blade material to the rub shroud surface can precipitate a buildup of material which causes blade wear. This mechanism is not fully understood.

Commonly used rub materials are a cast high-temperature cobalt base alloy, and sintered NiAl and NiCrAlY powders (Ref. 1). For long-term operation these materials must be cooled, by impingement and film cooling techniques, to temperatures in the range of $1311 \mathrm{~K}\left(1900^{\circ} \mathrm{F}\right)$. Nevertheless, thermal stress cracking, loss of dimensional stability, and erosion remain current problems. The cooling requirement in terms of engine airflow can be as high as $1 \%$ to $2 \%$, depending on the turbine-inlet temperature. This high cooling penalty is one reason why ceramic rub materials, which require less cooling, are being developed.

The use of ceramic material allows higher rub shroud surface temperatures. Further, ceramics require less cooling air. The potential cooling airflow reduction is indicated in Fig. 47 , which shows for a $1811 \mathrm{~K}\left(2800^{\circ} \mathrm{F}\right.$ ) turbine-inlet temperature and a surface temperature of $1700 \mathrm{~K}\left(2600^{\circ} \mathrm{F}\right)$, that the cooling air requirement is significantly less than when the surface temperature must be held to $1366 \mathrm{~K}\left(2000^{\circ} \mathrm{F}\right) ; \mathrm{Fig}$. 47 indicates a reduction from $2.1 \%$ to $0.3 \%$ of engine flow (Ref. 52 ).

An advanced ceramic/metal shroud being developed (Ref. 53) is shown in Fig. 48 . It is produced by thermal spraying ceramic/metal layers on a metal substrate of a heat resistant metal alloy. The first step in the process is to spray a $0.127-\mathrm{mm}(0.005-\mathrm{in}$.) coating of $\mathrm{NiCrAlY}$ on the metal substrate. This is followed by a layer composed of $60 \% \mathrm{CoCrAlY}$ and $40 \%$ yttria stabilized zirconia $\left(\mathrm{ZrO}_{2}\right)$. Next come successive layers of $30 \% \mathrm{CoCrAlY} / 70 \% \mathrm{ZrO}$, and $15 \% \mathrm{CoCrAlY} / 85 \% \mathrm{ZrO}_{2}$. Finally, the last layer, the one exposed directly to the turbine gas, is $100 \% \mathrm{ZrO}{ }_{2}$

This graded layer system provides a gradual change in thermal expansion coefficient and mitigates the large thermal expansion difference between the metal substrate and the ceramic layer next to the hot gas stream. Experimental studies (Ref. 53) show that graded layer ceramic material has adequate erosion resistance at $1588 \mathrm{~K}\left(2400^{\circ} \mathrm{F}\right.$ ) surface temperature. Encouraging thermal fatigue improvement is also reported but this remains the major problem. In this regard, an analytical study of the thermal stresses during engine acceleration (takeoff) by Ref. 54 indicates that the ceramic layer is probably subjected to excessive tensile stresses under this transient operati.ü condition.

\section{CONCLUDING REMARKS}

Modern gas turbines contain a multiplicity of sealing locations; a large engine may have over 50 major dynamic sealing locations; in addition, sealing is necessary at vane pivots, flanges, duct joints, and blade roots. As pointed out in the discussion, sealing can have a marked effect on engine efficiency, performance retention, thrust, compressor pressure ratio, and compressor stall margin. Much of the performance retention problem in modern engines is caused by increases in sealing clearances, with blade tip wear in the compressor and turbine being a major contributor. Improved sealing significantly increase air superiority mission radius and maximum dash distance. Also maximum thrust can be improved significantly. The performance deterioration trends suggest a sealing problem that will worsen as engine designs advance to even higher pressures and temperatures. Small engines remain a particularly challenging problem because leakage is inherently more detrimental to efficiency.

There are three general approaches to increasing sealing effectiveness, these are:

(1) Improved clearance control (reducing clearances and minimizing the amount of rubbing)

(2) Improved "abradable" materials

(3) Increased flow energy dissipation in labyrinth seals.

Of the three approaches, clearance control holds the most potential for improvement. To achieve clearance control improvements, we must know more about the relative displacement of the case and rotor, particularly under 
transient conditions, in modern engines. In advanced engines more attention must be given to engine stiffness, case roundness, case bending and to case/rotor dynamics of the total assembly. In this regard, more data are needed on various external and internal aerodynamic loads such as compressor surges. Accurate prediction of case/rotor displacements will require a large computational capability to treat the total assembly. Also, new approaches and concepts, such as active clearance control (by case cooling), will be needed to mitigate effects of case/rotor differential expansion. Improved "abradable" materials will provide some accommodation for eccentricity and out-of-roundness, but the clearance problem will probably not be solved solely with improvements in rub materials. In fact, the improved clearance control approach can be used to minimize rubbing and this, then, reduces the rub material problem.

The literature contains much data about blade clearance effects in compressors and turbines. Although there are variances between the published data, there is agreement that clearance changes cause a marked change in compressor and turbine efficiency. In particular, as fan and compressor clearances approach cero, the reported data indicate a very significant improvement in component efliciency. On the other hand, operation at very close clearances introduces a potential for high deterioration rates. Thus the solution requires maintaining close clearances for the time between normal overhauls. In the turbines the data show the clearance loss increasing with reaction rate; this trend suggests that advanced highly loaded turbines will have even greater losses than indicated in the current data. Thus advanced turbines will need to run with close clearances to avoid high losses.

Mathematical models for prediction of leakage over compressor and turbine blade tips are semiempirical expressions derived from cascade data; rotation effects are not included. There is a need for improved capability to predict clearance effects and for clearer insight regarding the significance of the many parameter which apparently affect tip leakage.

The ability of various engine companies to predict laby'rinth seal leakage is very good; the methods are semiempirical and involve a correlation with a large liumber of experiments. However, the large number and the impact of labyrinth seals on engine performance suggest studies should be made to identify unique labyrinth geometries which would reduce leakage; the potential gain is significant even if the leakage reduction is nominal. In regard to labyrinth seal theory, the thermodynamic process is well understool, but analy'sis from a lluid dynamic standpoint may shed light on means to increase the kinetic energy dissipation in the labyrinth cavities.

Experience indicates the necd for an improved compressor "abradable" material. The wear debris from this material should not stick on downstream air foils and should not pose a dust explosion problem in the high-pressure compressor. This "abradable" material also should produce little blade wear, be erosion resistant, and have an impermeable aerodynamically smooth surface.

Sealing over the tips of the high-pressure-turbine blade is a current problem, which will become most critical in advanced engines. Ceramic rub materials for the casc are needed to reduce cooling requirements and permit higher operating temperatures. These ceramic rub materials must be abradable (because close clearances will lead to rubs) and dimensionally stable to mitigate out-of-roundness and eccentricity effects.

As with the sealing of the primary-gas flow, sealing of the internal-gas flow is becoming more critical as engine's pressures and temperatures increase. There is a need for improved technology in the internal-flow system in regard to purging, heat transfer, and cooling flow control. In addition to improved labyrinth seals in the internal-flow system, mainshaft sealing with lower leakage and high pressure and speed capability would provide efficiency improvement.

\section{REFERENCES}

1. Mahler, F. H., Advanced Seal Technology. PWA-4372, 1972 (AD-739422).

2. Dugan, J. F., McAulay, J. E., Reynolds, T. W., and Strack, W. C., Fuel-Conservative Engine Technology. NASA SP-381, Aeronautical Propulsion, pp. 157-190, 1975.

3. Stern, J. A., Aircraft Propulsion. A Key' to Fuel Conservation: An Aircraft Manufacturer's View. SAE Paper 760538 , May 1976.

4. Legassie, R. W. A., and Ordway, F. I., A Quick Look at the National Energy Plan. Astronautics \& Aeronautics, Vol. 15, No. 11, Nov. 1977 , pp. $28-35$.

5. Grayson, K., Improved Maintenance Practices - The Airlines' Contribution to Lower Ownership Costs. SAE Paper 760504, May 1976.

6. Sallee, G. P., Kruckenberg, H. D., and Toumey, E. H., Analysis of Turboform Engine Performance Deterioration and Proposed Follow-on Tests. NASA CR-134769, 1975.

7. Csavina, F. L., Performance Depreciation of Some Mlilitary Turbofan Engines. ALAA Paper 76-649, July 1976. 
8. Hawkins, R. M. and McKibbin, A. H., Development of Compressor End Seals Stator Interstage Seals, and Stator Pivot Seals In Advanced Air Breathing Propulsion Systems, Part I: Screening Studies and Analysis. NASA CR-72819, 1970 .

9. Alford, J. S., Labyrinth Seal Designs Have Benefitted from Development and Service Experience. SAE Paper 710435,1971 .

10. Moore, A., Gas Turbine Engine Internal Air Systems - A Review of the Requirements and the Problems. ASME Paper 75-WA/GT-1, Nov. 1975.

11. Paladini, W., Static and Rotating Air/Gas Seal Evaluation. CW-WR-70-124F, 1971 (AD-730361).

12. Povinelli, Valentine P., Jr., Current Seal Designs and Future Requirements for Turbine Engine Seals and Bearings. Journal of Aircraft, Vol. 12, no. 4, April 1975, pp. 266-273.

13. Lynwander, P., Development of Helicopter Engine Seals. LYC-73-48, Nov. 1973. NASA CR-134647, 1973.

14. Schweiger, F. A., The Performance of Jet Engine Contact Seals. Lubrication Engineering, Vol. 19, June 1963, pp. $232-238$

15. Gray, D. E., and Dugan, J. F., An Early Glimpse at Long Term Subsonic Commercial Turbofan Technology Requirements - Fuel Conservation. AlAA Paper 75-1207, Sep. 1975.

16. Lakshminarayana, B. and Horlock, J. H., Tip-Clearance Flow and Losses for an Isolated Compressor Blade. University of Liverpool, R \& M No. 3316, 1963.

17. Dean, R. C., Jr., Secondary Flow in Axial Compressors. MIT Thesis, 1954. Also MIT Report 54-10-T, 1954.

18. Rains, D. A., Tip Clearance Flows in Axial Flow Compressors and Pumps. California Institute of Technology Report No. 5, 1954.

19. Lakshminarayana, B., Methods of Predicting the Tip Clearance Effects in Axial Flow Turbomachinery. ASME Paper 69-WA/FE-26, Nov. 1969.

20. Egli, A., The Leakage of Steam Through Labyrinth Seals. ASM1E Trans., Vol. 57, No. 3, Apr. 1935, pp. 115122.

21. Dobek, L. J., Labyrinth Seal Testing For Lift Fan Engines. NASA CR-121131, 1973.

22. Zuk, J., Dynamic Sealing Principles. NASA TM X-71851, 1976.

23. Stocker, H. L., Cox, D. M., and Holle, G. F., Acrodynamic Performance of Conventional and Advanced Design Labyrinth Seals With Solid-Smooth, Abradable, and Honeycomb Lands, Detroit Diesel Allison, EDR 9339, NASA CR-135307, Dec. 1977. (To be published.)

24. Moore, R. D. and Osborn, W. M., Effects of Tip Clearance on Overall Performance of Transonic Fan Stage With and Without Casing Treatment. NASA TM X-3479, 1977.

25. Jefferson, J. L. and Turner, R. C., Some Shrouding and Tip Clearance Effects in Axial Flow Compressors. International Shipbuilding Progress, Vol. 5, No. 42, Feb. 1958, pp. 78-101.

26. White, J. L. and Beste, D. L. Nastran Applications to Aircraft Propulsion Systems. NASA TM X-3278, NASTRAN: USER'S EXPERIENCES, pp. 91-104, 1975.

27. Benstein, E. H., Small Flying Engines Are Different - Aircraft Gas Turbine Design. ALAA Paper 74-1185, Oct. 1974.

28. Holman, F. F., Kidwell, J. R., and Ware, T. C., Small Axial Compressor Technology Program. NASA CR-134827, Vol. 1, 1976.

29. Klassen, H. A., Wood, J. R., and Schumann, L. F., Experimental Performance of a 13.65 Centimeter-TipDiameter Tandem-Bladed Sweptback Centrifugal Compressor Designed for a Pressure Ratio of 6 . NASA TP-1091, 1977.

30. Klassen, H. A., Wood, J. R., and Schumann, L. F., Experimental Performance of a 16. 10-Centimeter-TipDiameter Sweptback Centrifugal Compressor Designed For a 6:1 Pressure Ratio. NASA TM X-3552, 1977.

31. Holeski, D. E. and Futral, S. M., Jr., Effect of Rotor Tip Clearance on the Performance of a 5-Inch SingleStage Axial-Flow Turbine. NASA TM X-1757, 1969. 
32. Kofskey, M. G., Experimental Investigation of Three Tip-Clearance Configurations Over a Range of Tip Clearance Using a Single-Stage Turbine of High Hub-to Tip-Radius Ratio. NASA TM X-472, 1961.

33. Szanca, E. M., Behning, F. P., and Schum, H. J., Research Turbine for High-Temperature Core Engine Application. II - Effect of Rotor Tip Clearance on Overall Performance. NASA TN D-7639, Apr. 1974.

34. Haas, J. E. and Kofskey, M. G., Cold-Air Performance of a 12.766-Centimeter-Tip-Diameter Axial-Flow Cooled Turbine. IIl - Effect of Rotor Tip Clearance on Overall Performance of a Solid Blade Configuration. NASA TP-1032, 1977.

35. Futral, S. M., Jr. and Holeski, D. E., Experimental Results of Varying the Blade Shroud Clearance in a 6.02Inch Radial-Inflow Turbine. NASA TN D-5513, 1970.

36. Beyerly, W. R. and Sweeney, J. G., Life Cycle Fuel Consumption of Commercial Turbofan Engines. AIAA Paper 76-645, July 1976.

37. Adamson, G. P., Development Progress-New Transport Engines, JT9D. SAE Paper 710419, May 1971.

38. Aarnes, M. N. and White, J. L., Propulsion System and Airframe Structural Integration Analysis. AIAA Paper 75-1310, Sep. 1975.

39. Gunter, E. J., Jr., Dynamic Stability of Rotor-Bearing Systems. NASA SP-113, 1966.

40. Kirk, R. G. and Gunter, E'. J., Effect of Support Flexibility and Damping on the Dynamic Response of a Single Mass Flexible Rotor in Elastic Bearings. NASA CR-2083, 1972.

41. Tessarik, J. M., Flexible Rotor Balancing by Influence Coefficient Method - Multiple Critical Speeds with Rigid or Flexible Supports. NASA CR-2553, 1975.

42. Alwang, W. G. and Kinchen, B., Internal Running Clearance Measurements in Gas Turbines Using High Energy $\mathrm{X}$-Radiography, In "Advances in Test Measurement, Vol. 12; Ed. by B. Washburn, Instrument Society of America, 1975, pp. 339-34s.

43. Ford, M. J., Hildebrand, J. R., and Prosser, J. C., Design, Fabrication and Demonstration of a Miniaturized Tip Clearance Measuring Device. PWA-FR-6447, 1974.

44. Osborn, W. M., Lewis, G. W., Jr., and Heidelberg, L. J., Effect of Several Porous Casing Treatments on Stall Limit and on Overall Performance of an Axial-Flow Compresser Rotor. NASA TN D-6537, 1971.

45. Bailey, E. E. and Voit, C. H., Some Observations of Effects of Porous Casings on Operating Range of a Single Axial-Flow Compressor Rotor. NASA TN X-2120, 1970.

46. Prince, D. C., Jr., Wisler, D. C., and Hilvers, D. E., Study of Casing Treatment Stall Margin Improvement Phenomena - For Compressor Rotor Blade Tips Compressor Blades Rotating Stalls. NASA CR-134552, 1974.

47. Bailey, E. E., Effects of Grooved Casing Treatment on the Flow Range Capability of Single-Stage Axial-Flow Compressor. NASA TM X-2459, 1972.

48. Bill, R. C. and Shiembob, L. T., Friction and Wear of Sintered Fiber-Metal Abradable Seal Materials. NASA TM X-73650, 1977 .

49. Burton, R. A., Kilaparti, S. R., and Hechmann, S. R., Mlodeling of Turbine Blade Tip Contact. ASME Paper 75-WA/GT-14, Nov. 1975.

50. Barber, J. R., Thermoelastic Instabilities in the Sliding of Conforming Solids. Proceedings of the Royal Society, Series A, Vol. 312, No. 1510, Sep. 1969, pp. 351-394.

51. Burton, R. A., Nerlikar, V., and Kilaparti, S. R., Thermoelastic Instability in a Seal-Like Configuration. Wear, Vol. 24, 1973, pp. 177-188.

52. Schlike, P. W., Advanced Ceramic Seal Program (Phase I) PWA 6635, 1974 (AD 781004).

53. Shiembob, L. T., Development of a Plasma Sprayed Ceramic Gas Path Seal for High Pressure Turbine Applications. NASA CR-135183, 1977.

54. Taylor, C. M., 'Ihermal Stress Analysis of a Graded Zirconia/Metal Gas Path Seal System for Aircraft Gas Turbine Engines, NASA TM X-73658, 1977. 
TABLE I. - TYPICAL DIRECT OPERATING

COSTS (FROM REF. 3)

\begin{tabular}{|l|r|r|r|}
\hline \multirow{2}{*}{ Item } & \multicolumn{3}{|c|}{ Fuel price } \\
\cline { 2 - 4 } & 20 \%/gal & 35 ç/gal & 50\%/gal \\
\cline { 2 - 4 } & \multicolumn{3}{|c|}{ Direct operating cost, \% } \\
\hline Fuel & 31.5 & 44.6 & 53.5 \\
Depreciation & 25.5 & 20.6 & 17.3 \\
Maintenance & 19.0 & 15.4 & 12.9 \\
Crew & 18.2 & 14.7 & 12.3 \\
Insurance & 5.8 & 4.7 & 4.0 \\
\hline Change in DOC & ---- & 23.6 & 47.2 \\
\hline
\end{tabular}

TABLE II. - VIBRATIONS AFFECTING CLEARANCES

\begin{tabular}{|c|c|c|}
\hline Type & Exciting force & Comment \\
\hline 1. Rigid shaft whirl & Rotor unbalance & $\begin{array}{l}\text { Critical speed generally lower } \\
\text { than engine operating speed } \\
\text { and displacements are } \\
\text { typically small }\end{array}$ \\
\hline 2. Flexible shaft & Rotor unbalance & $\begin{array}{l}\text { Multiplane balancing technology } \\
\text { is available - implementation } \\
\text { needed }\end{array}$ \\
\hline $\begin{array}{l}\text { 3. Labyrinth seal whirl forces } \\
\text { (tip shrouded blades and } \\
\text { labyrinth seals with non- } \\
\text { uniform clearances) }\end{array}$ & $\begin{array}{l}\text { Nonaxisymmetric pressure in } \\
\text { labyrintin seal which causes } \\
\text { a force transverse to eccen- } \\
\text { tricity direction }\end{array}$ & Studies are needed \\
\hline $\begin{array}{l}\text { 4. Blade whirl forces } \\
\text { (shrouded and unshrouded } \\
\text { blades with nonuniform } \\
\text { clearances) }\end{array}$ & $\begin{array}{l}\text { Nonaxisymmetric blade load- } \\
\text { ing due to tip leakage } \\
\text { causes a force transverse } \\
\text { to eccentricity direction }\end{array}$ & Studies are needed \\
\hline 5. Rub induced whirl & $\begin{array}{l}\text { Frictional force of labyrinth } \\
\text { teeth or of blades rubbing } \\
\text { against case }\end{array}$ & Studies are needed \\
\hline
\end{tabular}




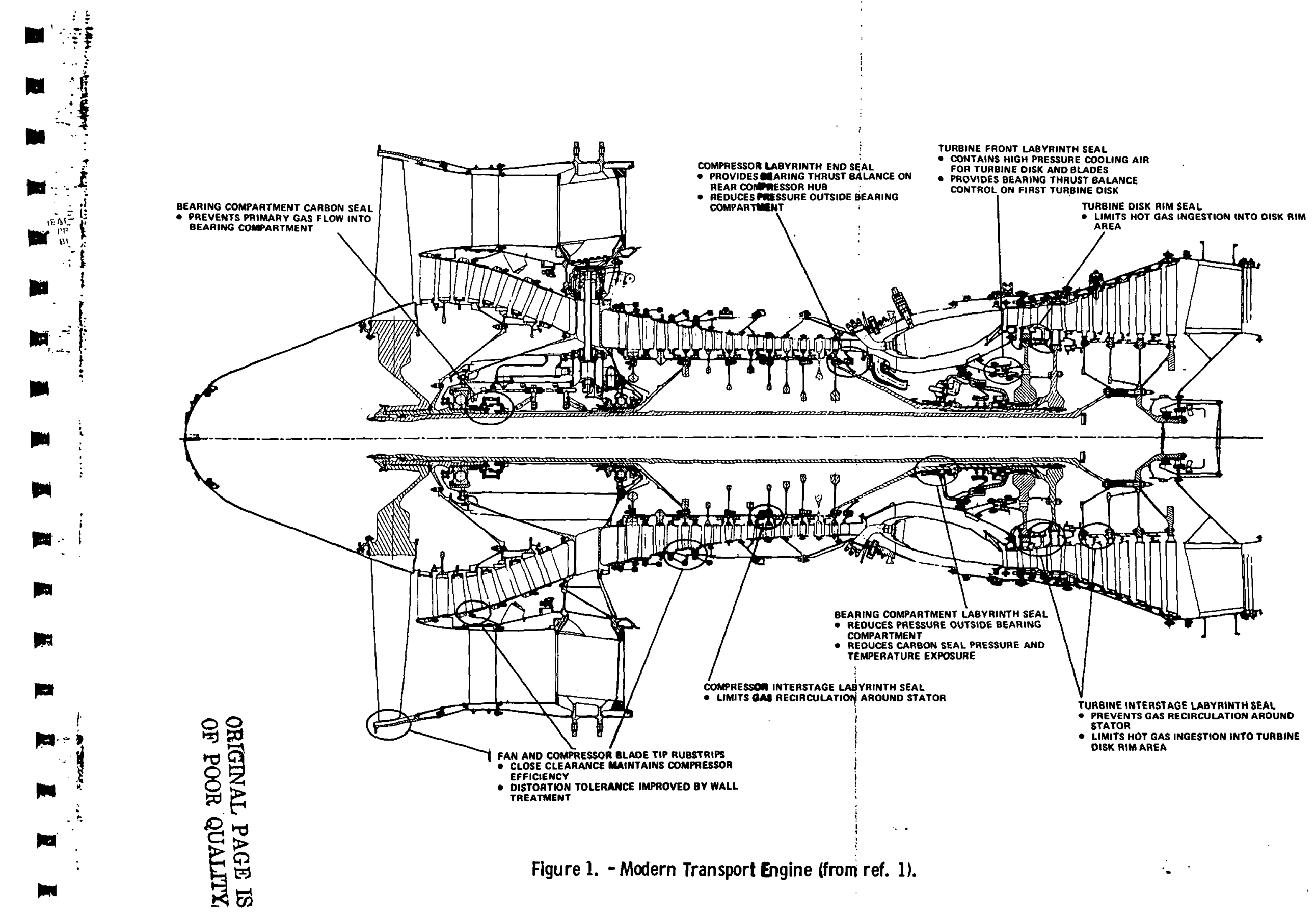




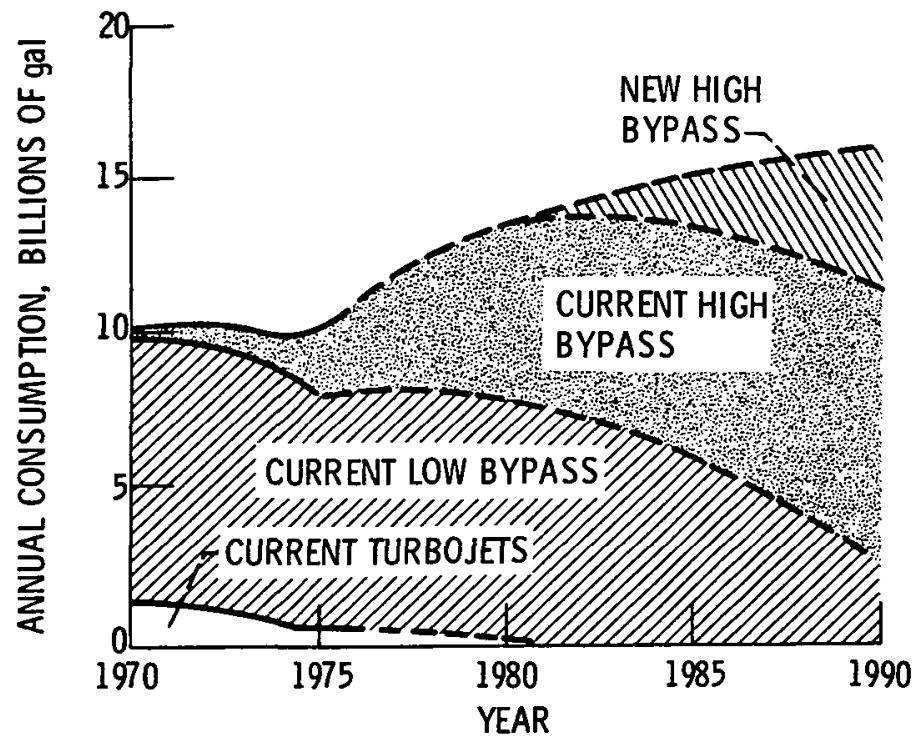

Figure 2 - U.S. airline fuel consumption by engine type (from ref. 2).

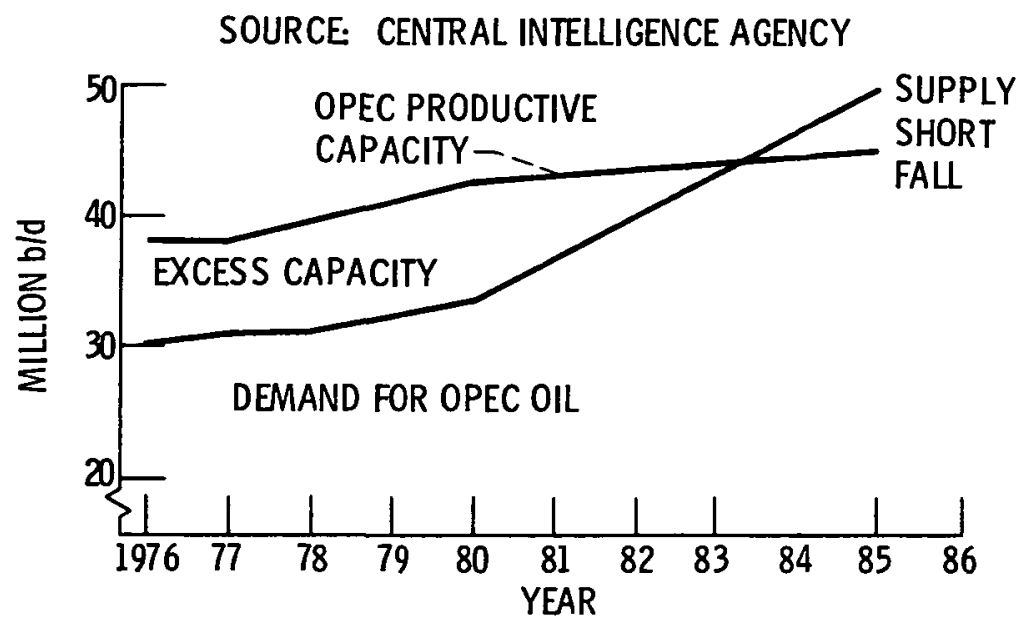

Figure 3. - OPEC oil, supplyldemand gap (from ref. 4). 


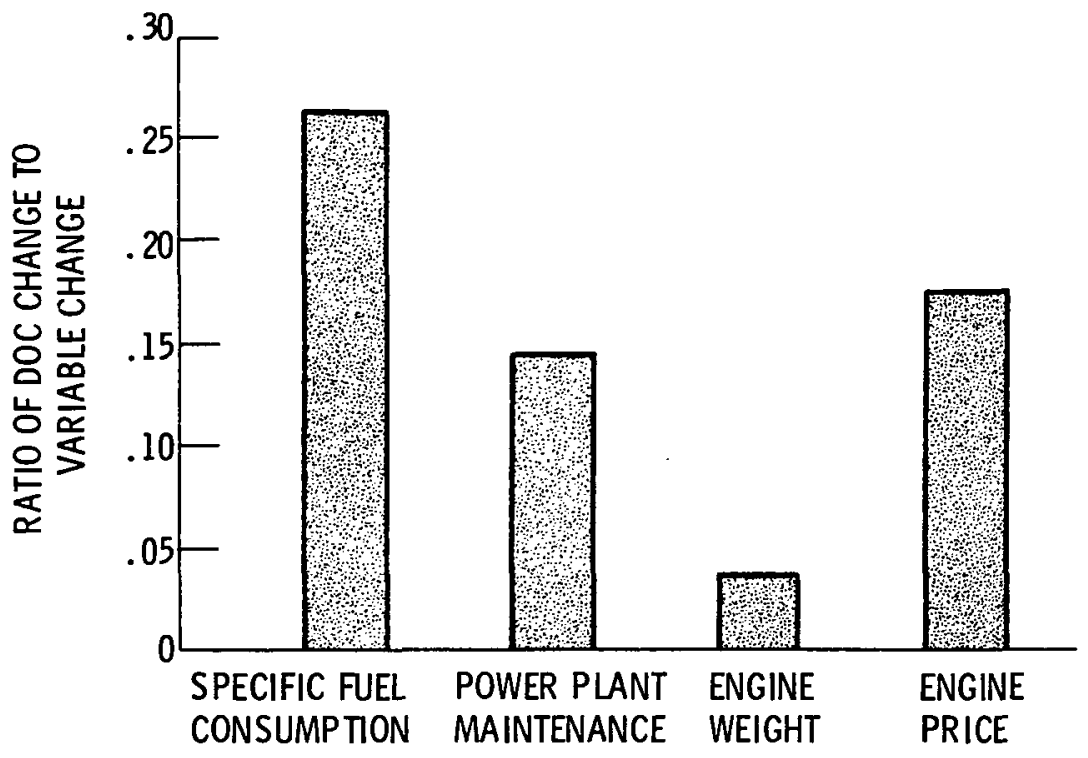

Figure 4. - Direct operating cost-sensitivity to propulsion system variable (from ref. 5).

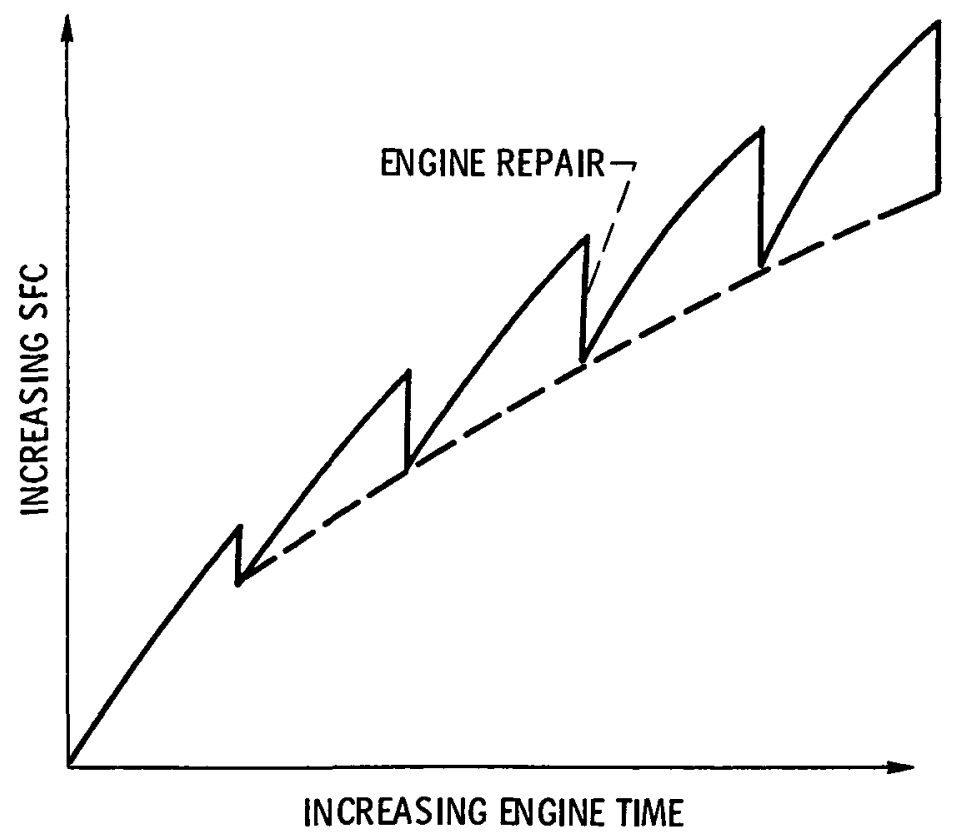

Figure 5. - Performance deterioration. 


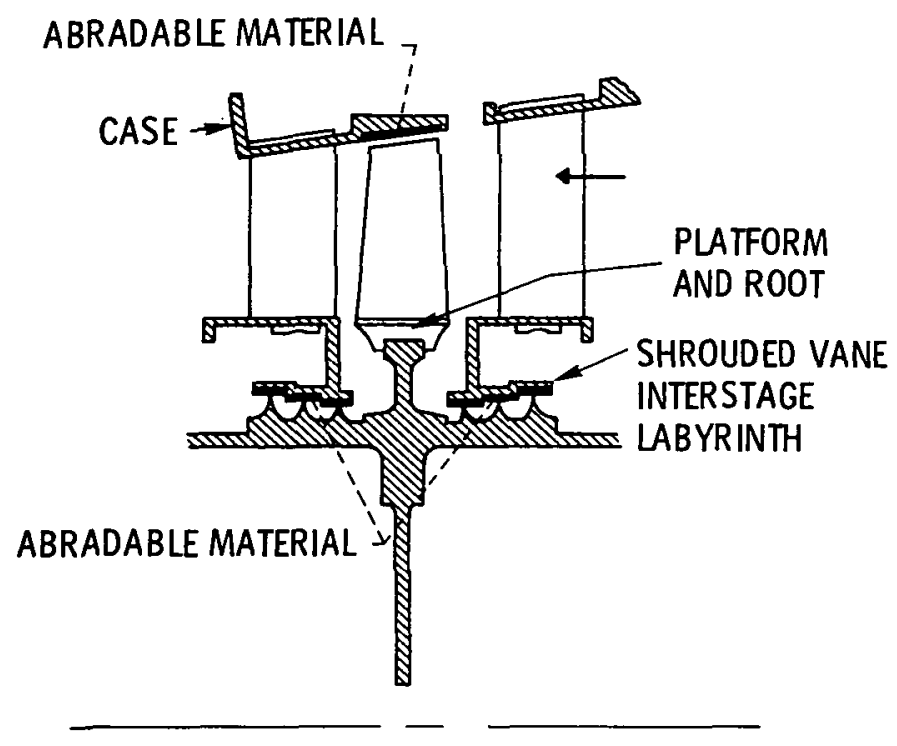

(a) BLADE TIP AND INTERSTAGE.

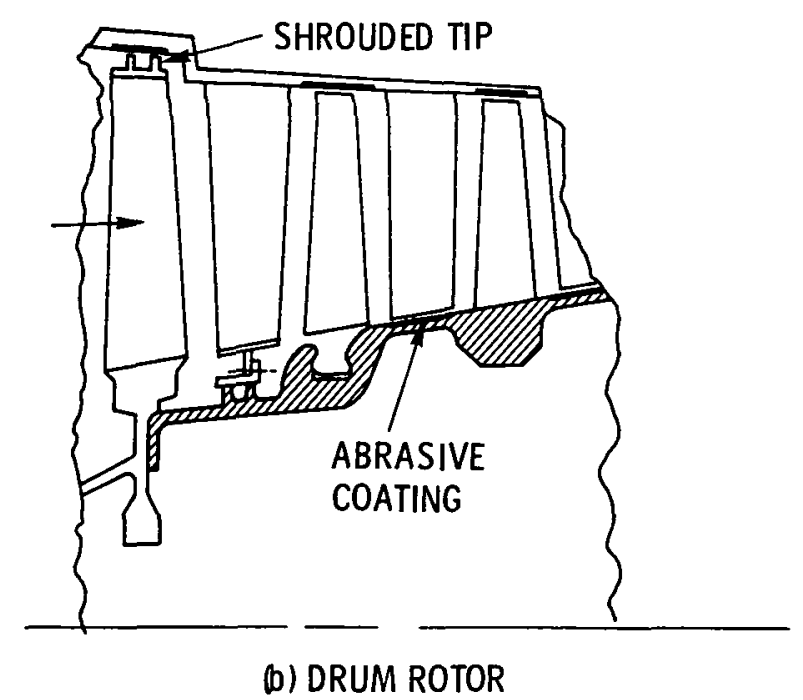

Figure 6. - Compressor sealing locations. 


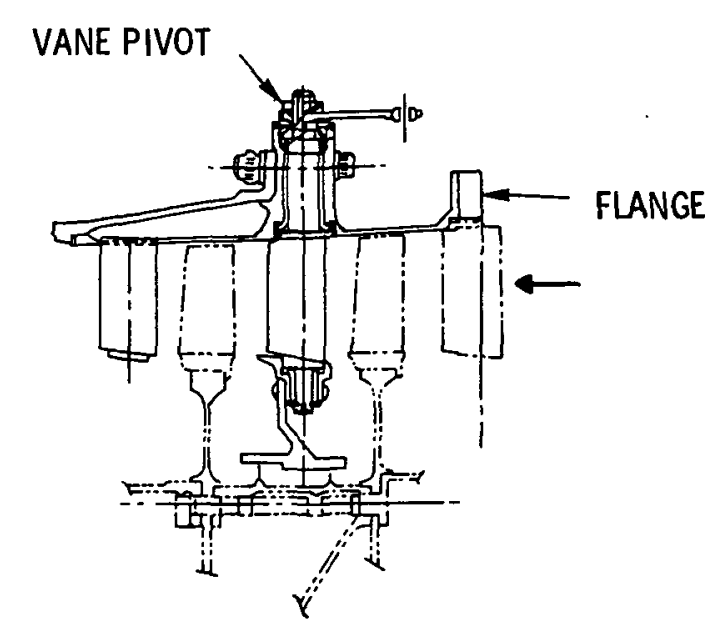

(c) VANE PIVOT AND FLANGE (REF. 1).

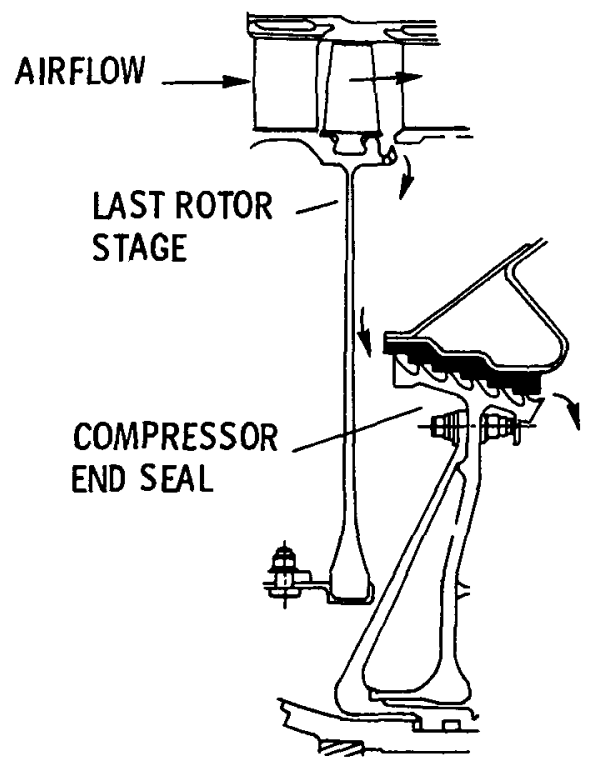

(d) COMPRESSOR DISCHARGE LABYRINTH SEAL (REF. 9).

Figure 6. - Concluded. 


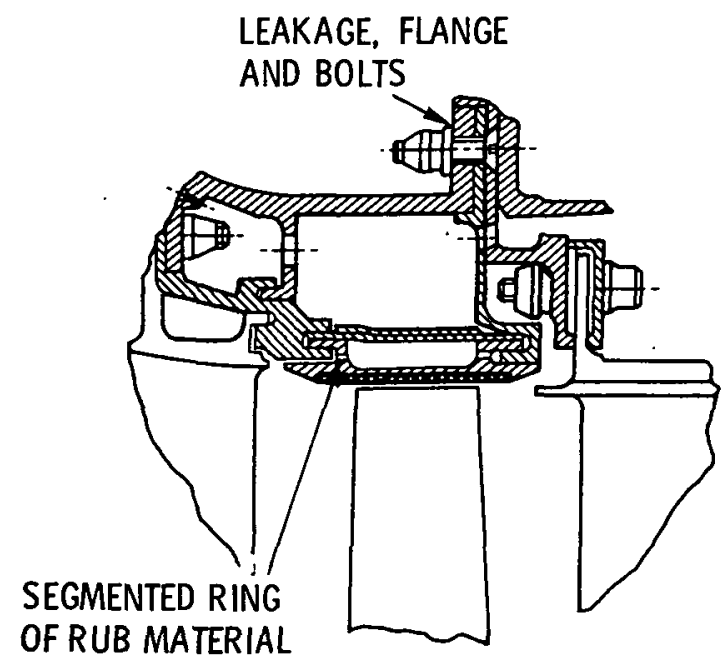

(a) BLADE TIP.

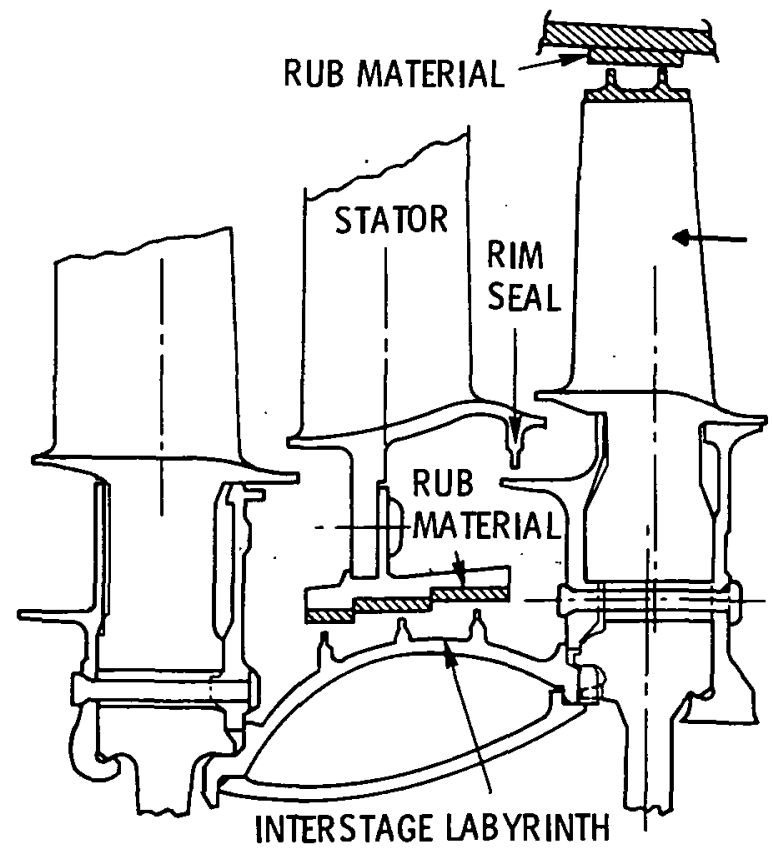

(b) SHROUDED ROTOR AND INTERSTAGE.

Figure 7. - Turbine sealing locations (ref. i). 


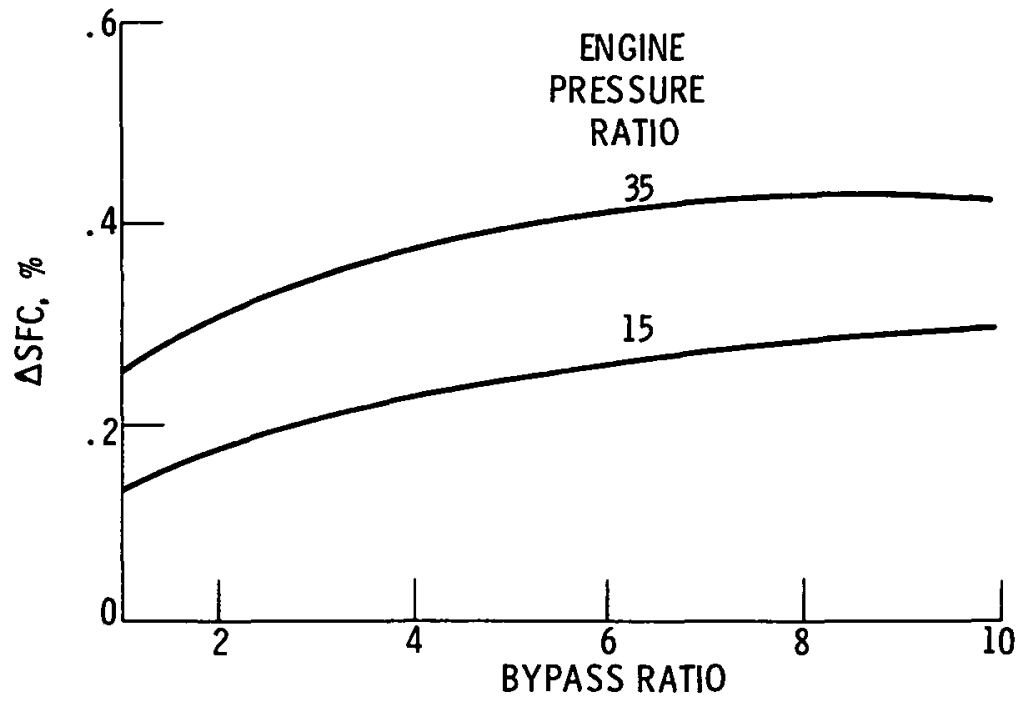

Figure 8. - Effect of one percentage point increase in engine bleed on SFC for various bypass ratios and for two pressure ratios (ref. 10 ). 


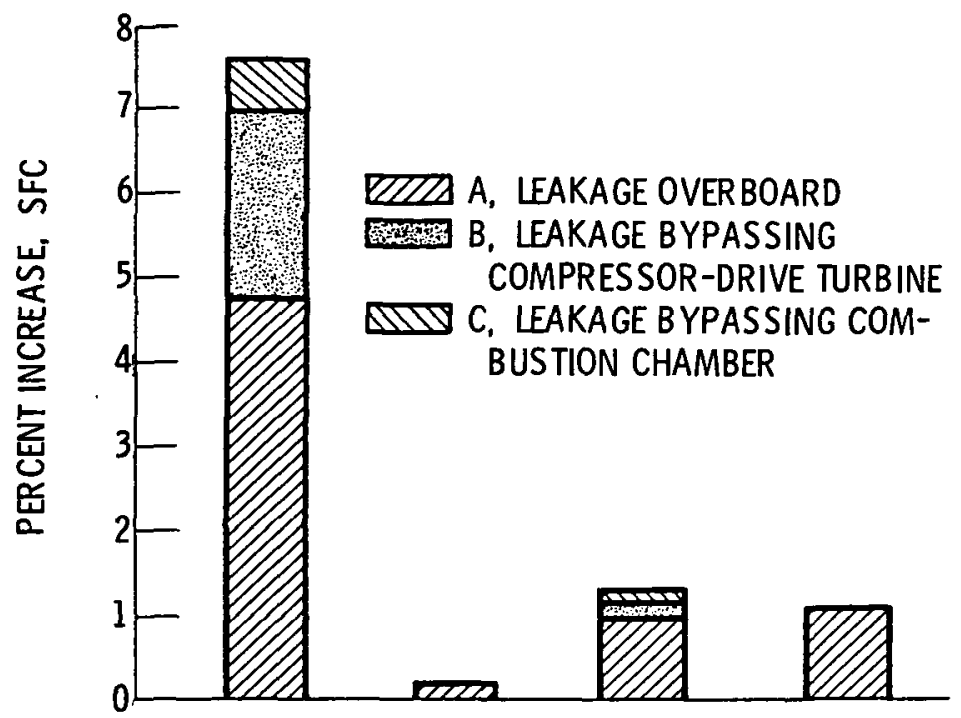

(a) SPECIFIC FUEL CONSUMPTION.

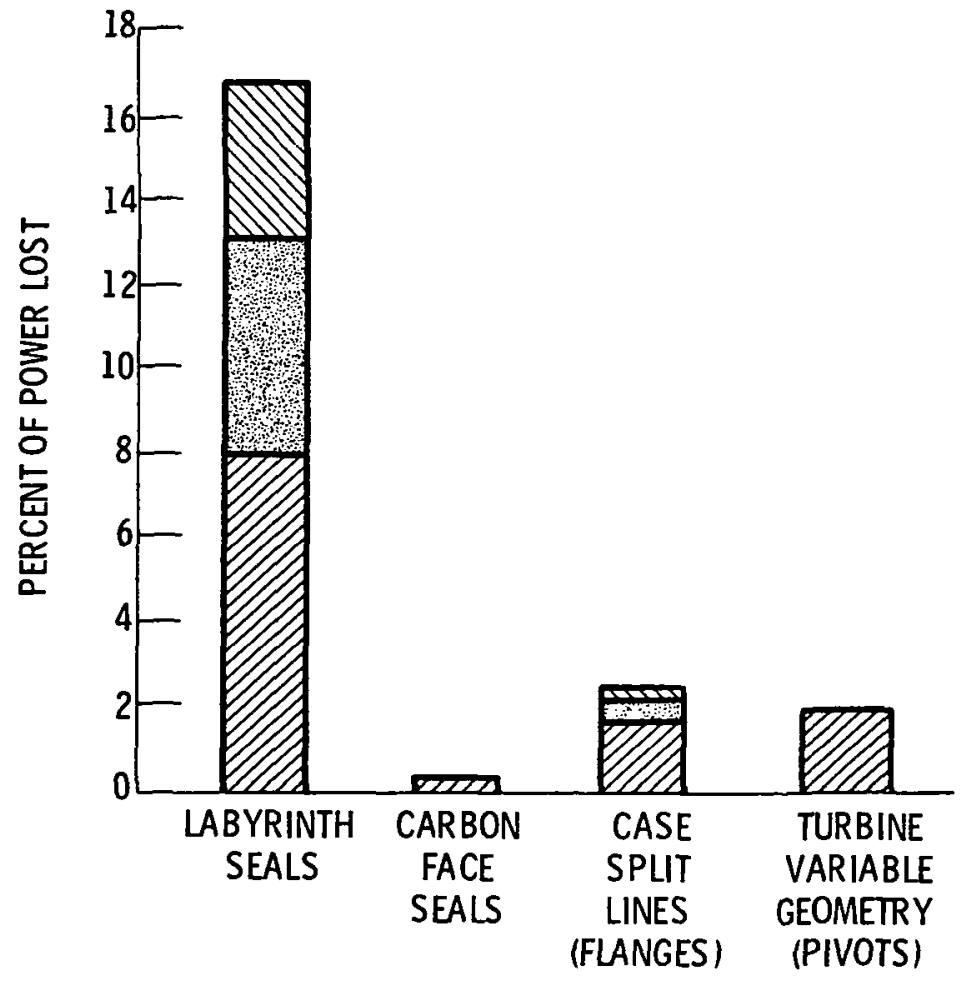

b) POWER.

Figure 9. - Calculated effects of seal leakage in a $2.3 \mathrm{~kg}$ per second ( $5 \mathrm{lbm}$ per sec) size engine (ref. 11$)$.

ORIGINAL PAGE IS OF POOR QUALT:S 


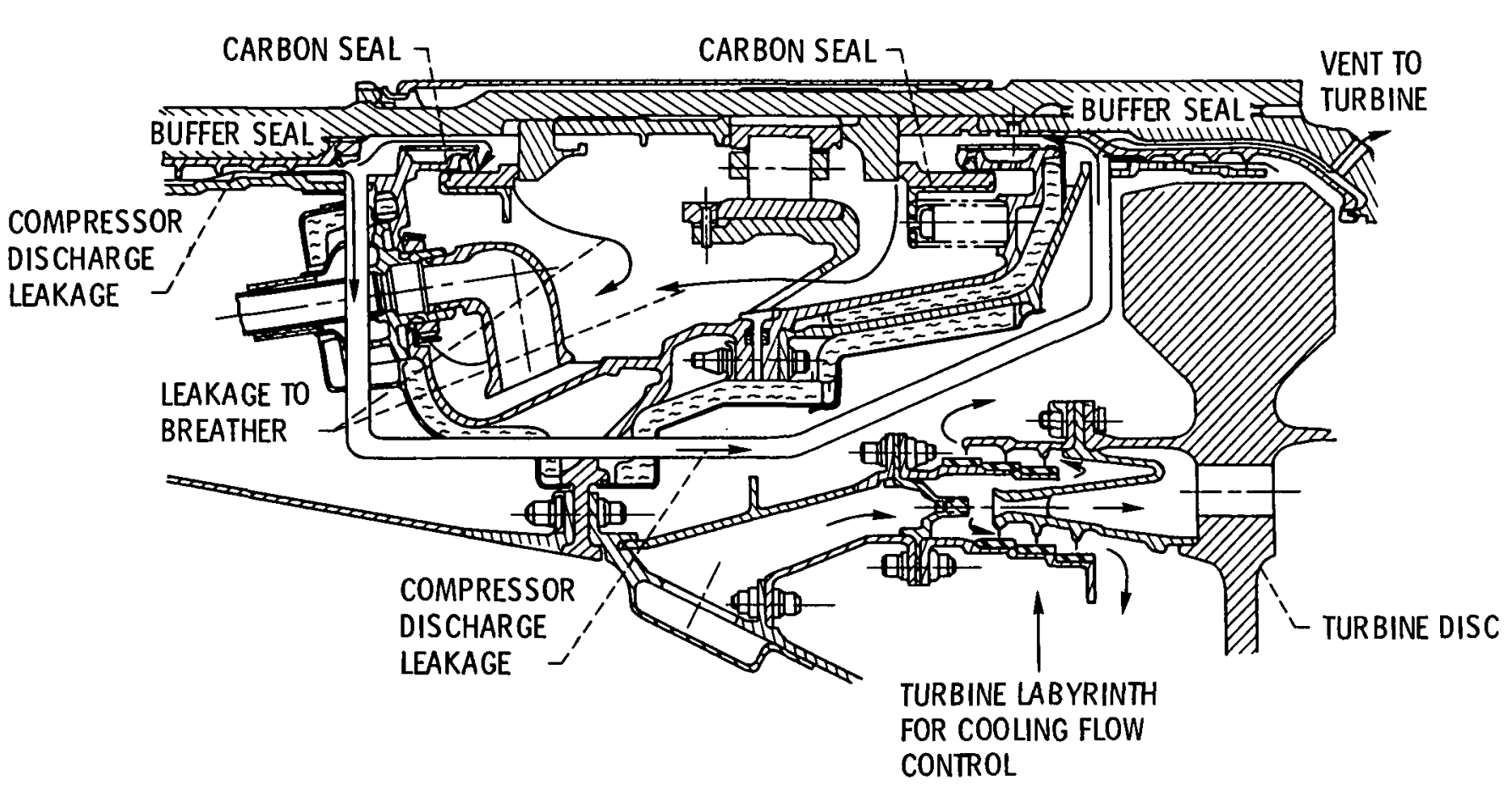

Figure 10. - Mainshaft and high pressure turbine labyrinth seal (from ref. 1). 


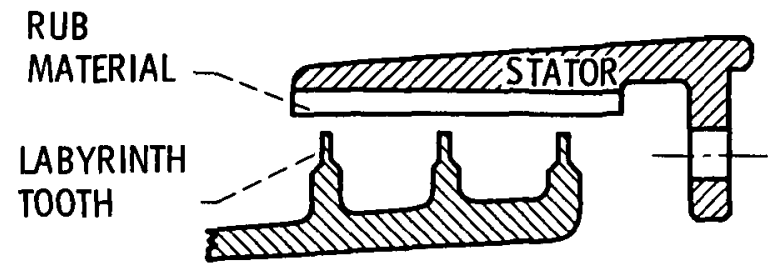

(a) STRAIGHT.

RUB

MATERIAL - TIZZT STATOR

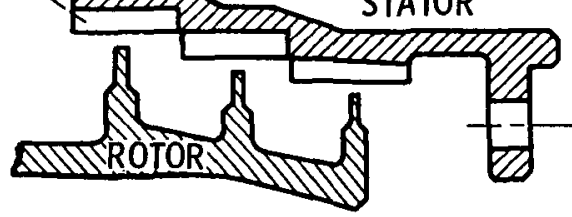

D) STEPPED.

Figure 11. - Labyrinth seal types.

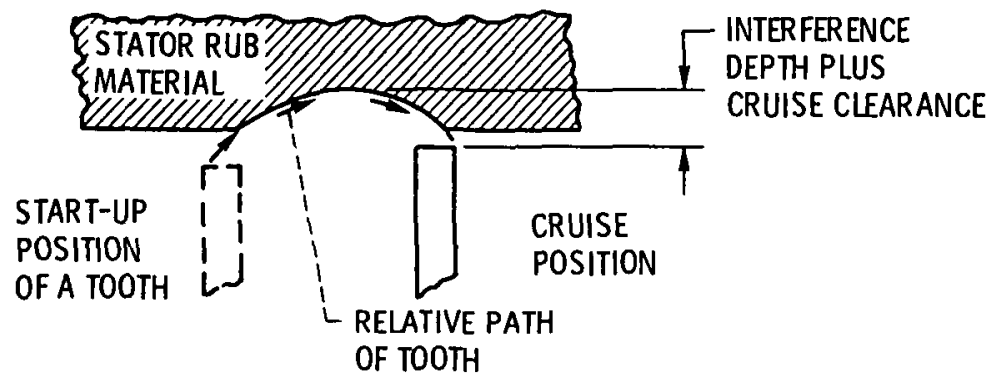

(a) RADIAL AND AXIAL MOTION.

TRANSIENT

PENETRATION

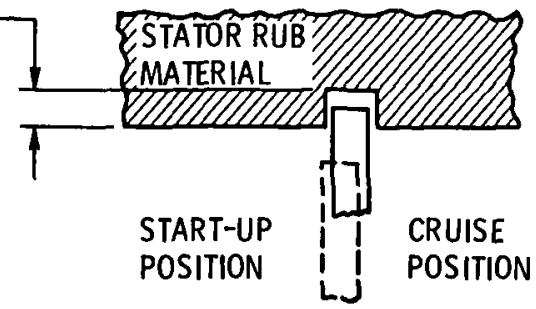

b) RADIAL MOTION, IDEAL INTERLOCKING.

Figure 12. - Labyrinth seal leakage areas for total interference wear to the stator. 


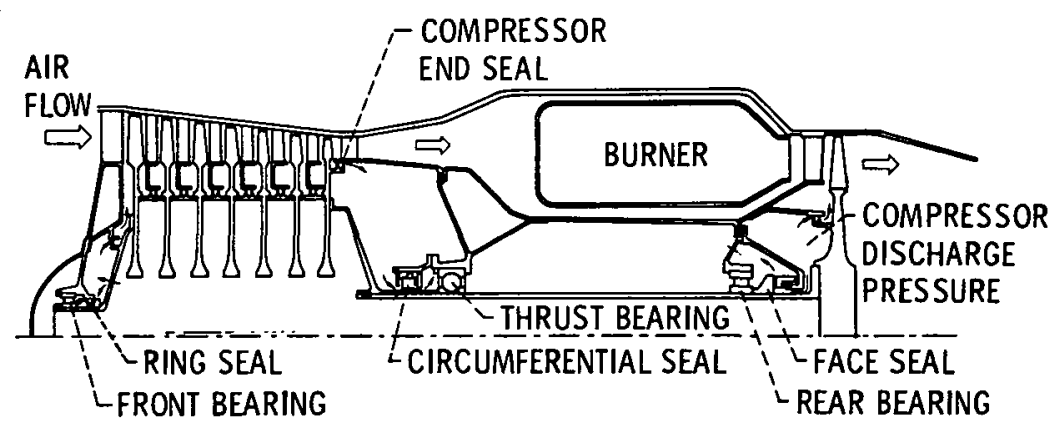

Figure 13. - Engine schematic showing main shaft seal locations.

ORIGINAL PAGE IS

OF POOR QUALITY 


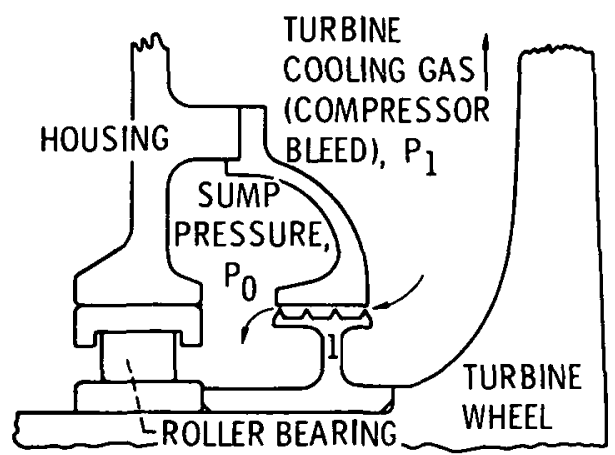

(a) Single labyrinth, early engines.

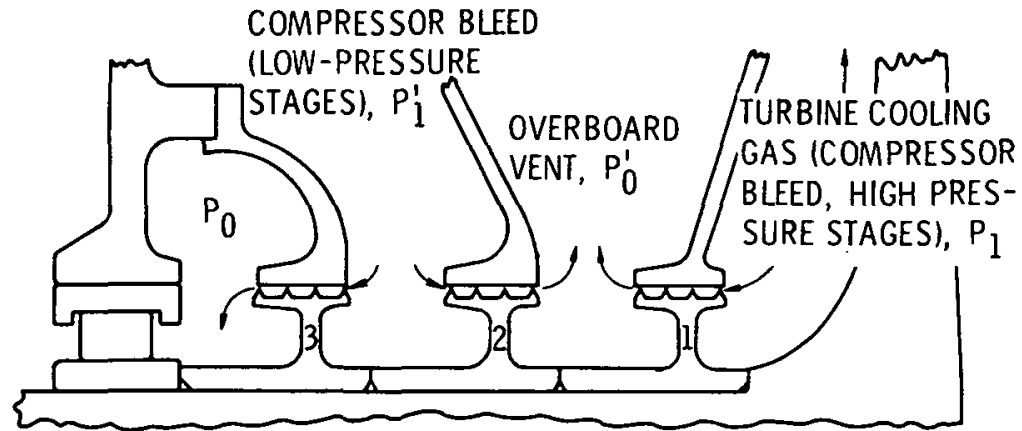

(b) Multiple labyrinth for high-temperature high-pressure turbine cooling gas.

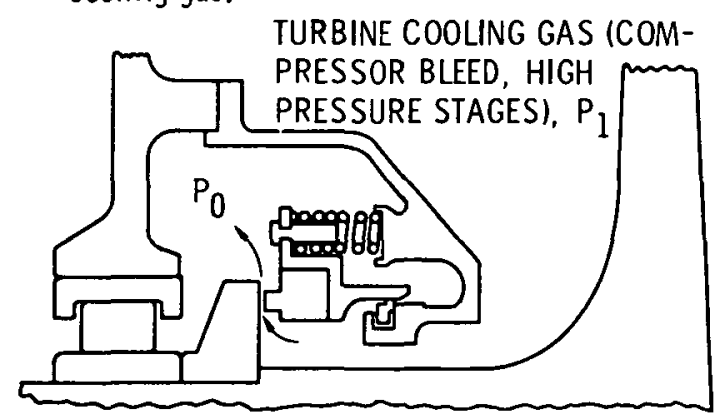

(c) Conventional face seal.

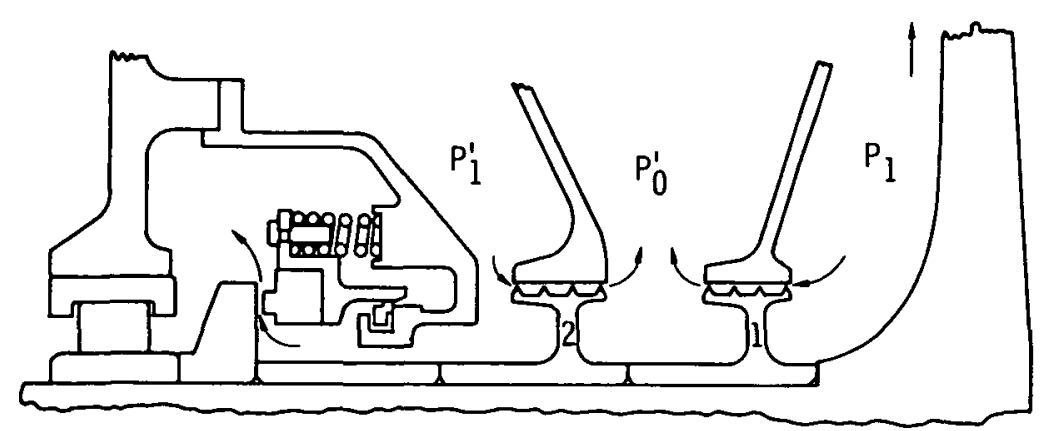

(d) Conventional face seal with labyrinth seal for high-temperature, high-pressure cooling gas.

CD-10293-28

Figure 14. - Seal systems. 


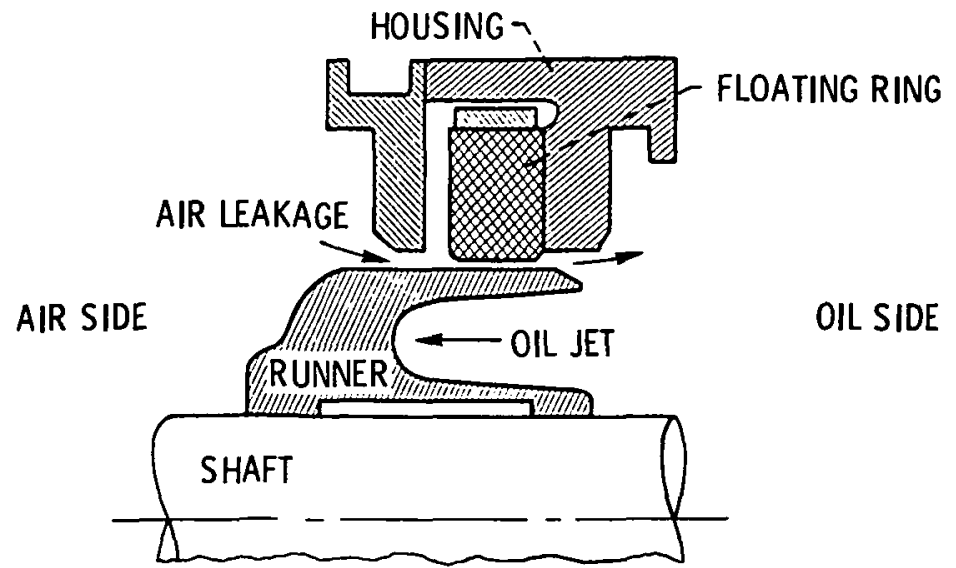

(a) FLOATING RING SEAL.

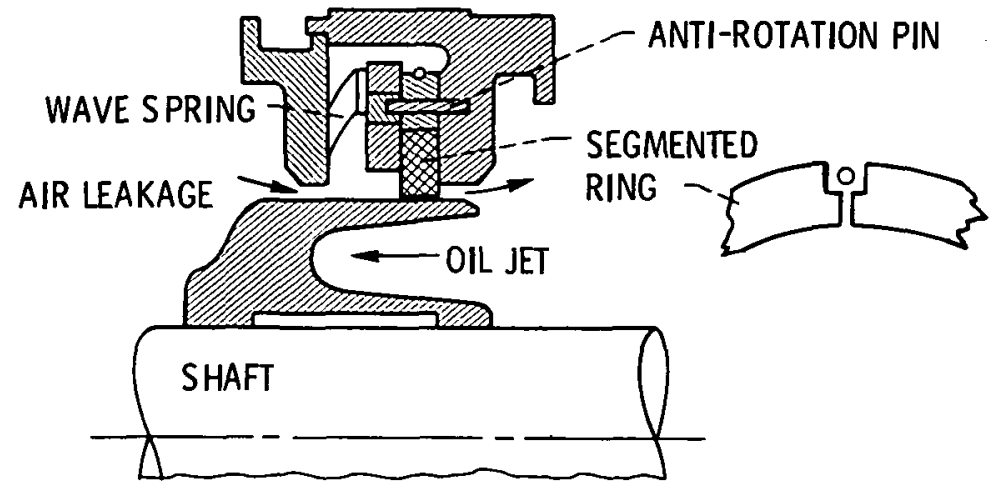

(b) UNBALANCED CIRCUMFERENTIAL SEGMENTED-RING SEAL.

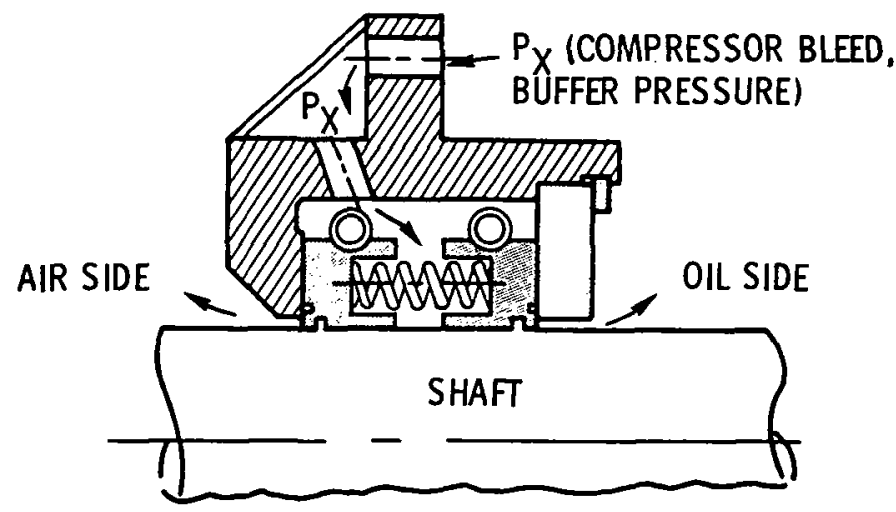

(c) PRESSURE BALANCED CIRCUMFERENTIAL SEAL, SEGMENTED-RING.

Figure 15. - Close clearance shaft seals, circumferential types.

ORIGINAL PAGE Ts OF POOR QUALITY 


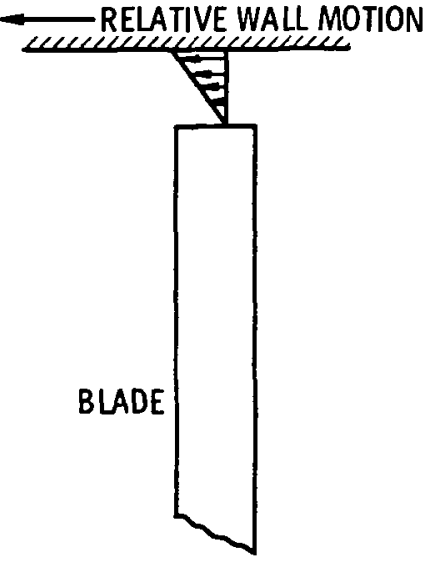

(a) COUETIE FLOW VELOCITY DISTRIBUTION DUE TO RELATIVE WALL MOTION.

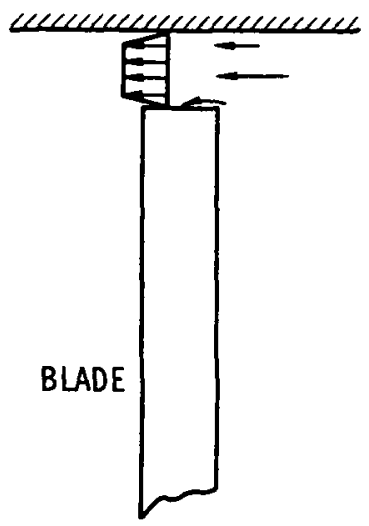

b) ORIFICE TYPE FLOW DUE TO PRESSURE DIFFERENCE.

Figure 16. - Flow velocity profiles.

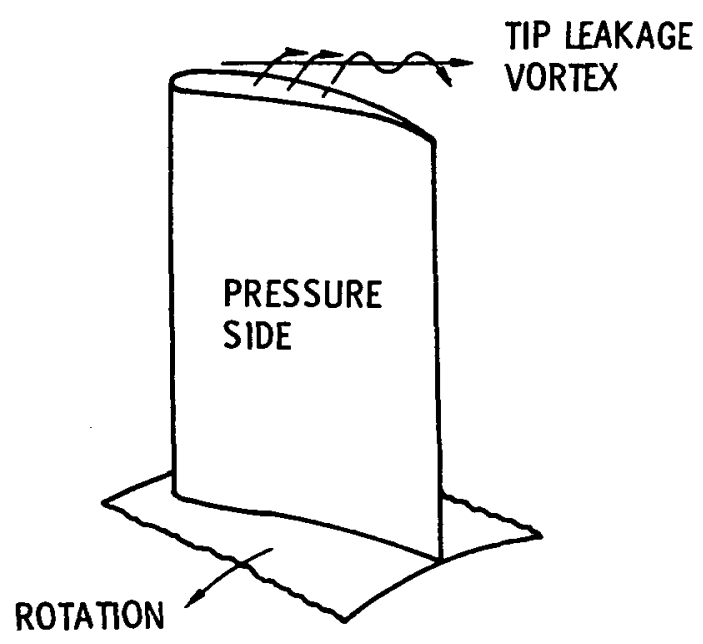

Figure 17. - Vortex formed by leakage over blade tip. 
CASE I.D.

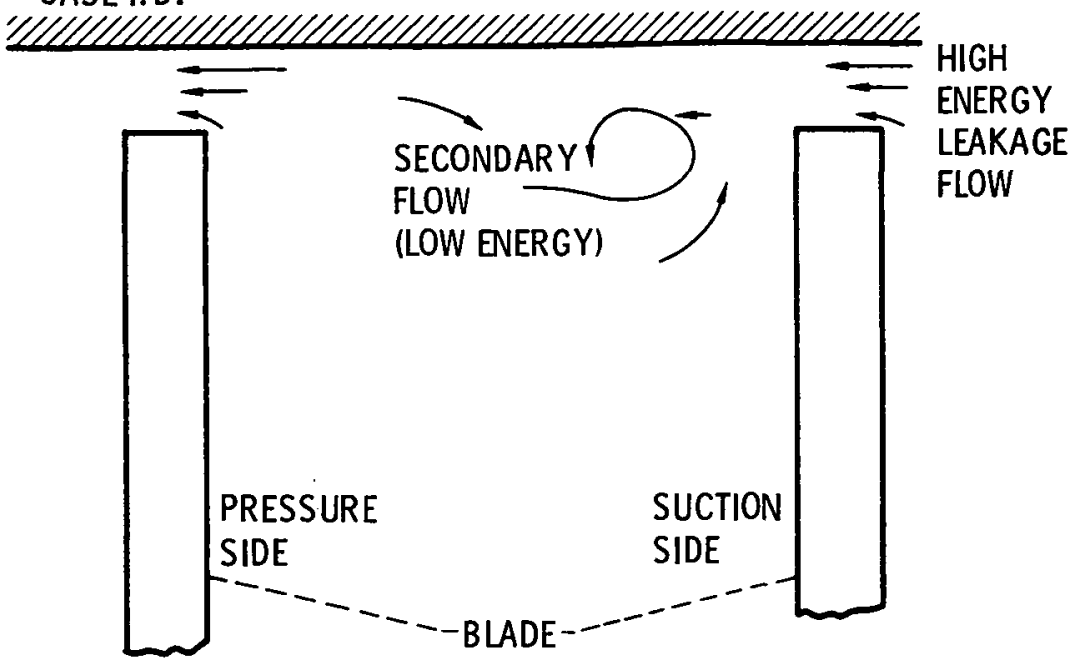

Figure 18. - Interaction of leakage and secondary flow.

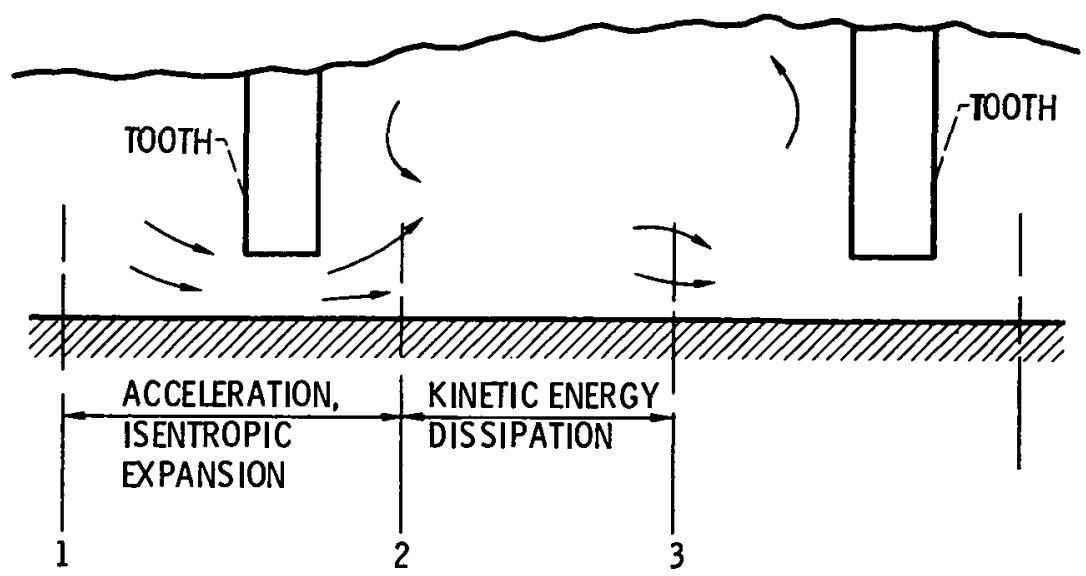

Figure 19. - Labyrinth seal, thermodynamic model.

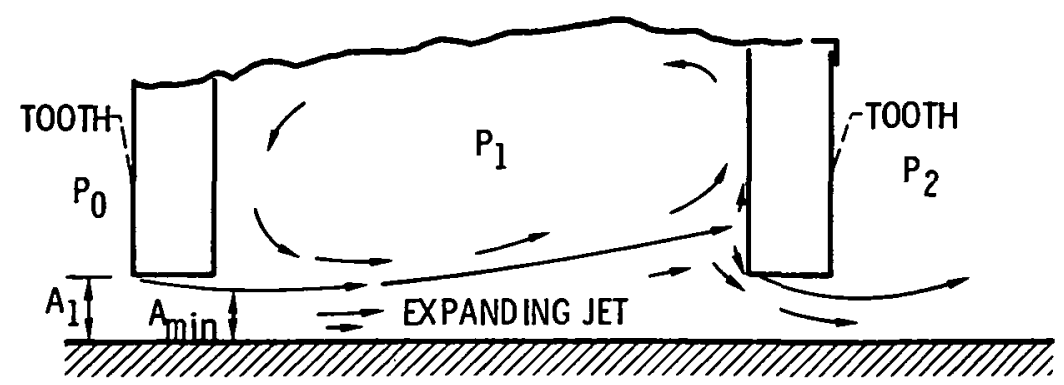

Figure 20 . - Labyrinth seal, fluid mechanic model illustrating the carryover effect. 


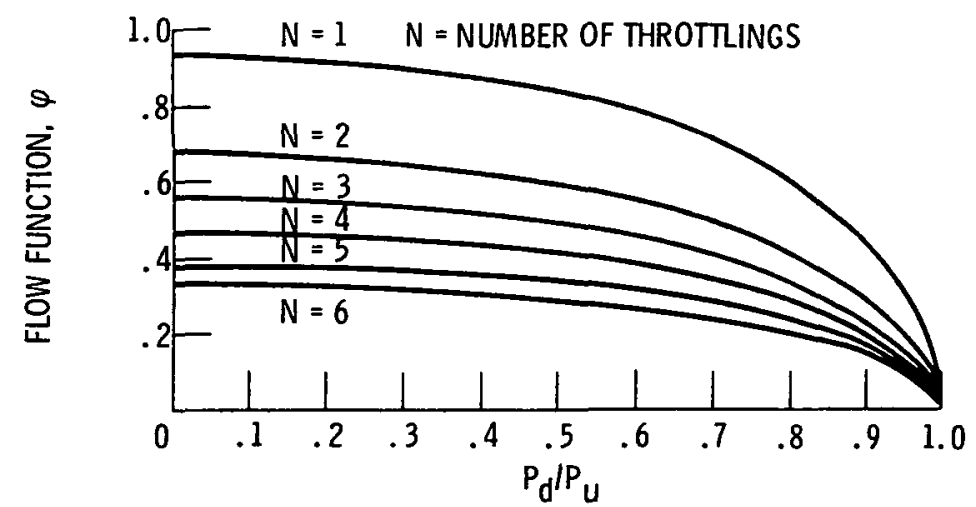

(a) FLOW FUNCTION.
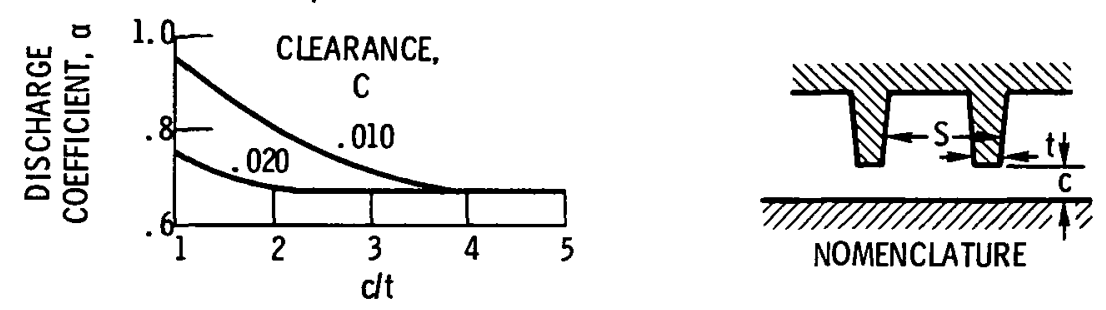

(b) DISCHARGE COEFFICIENT

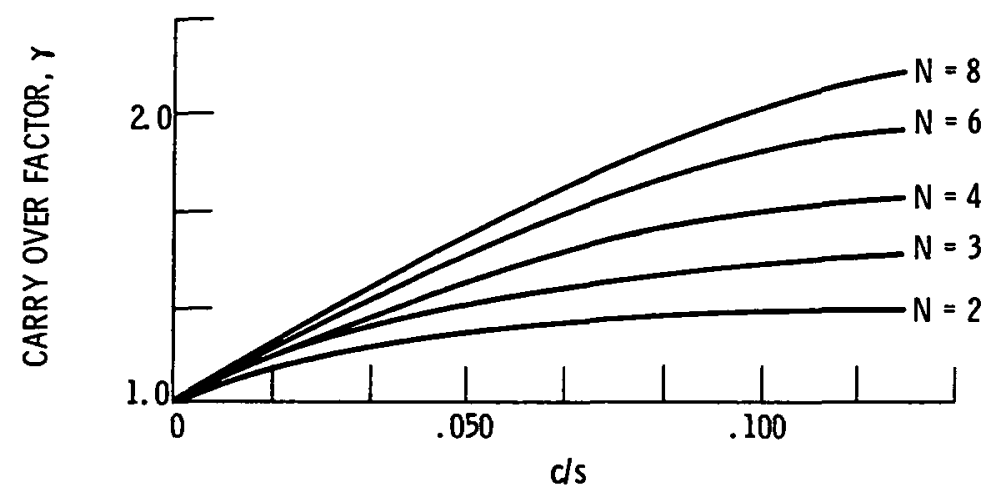

(c) CARR Y-OVER FACTOR.

Figure 21. - Plots of functions used in Egli labyr inth seal leakage equation (from ref. 22). 


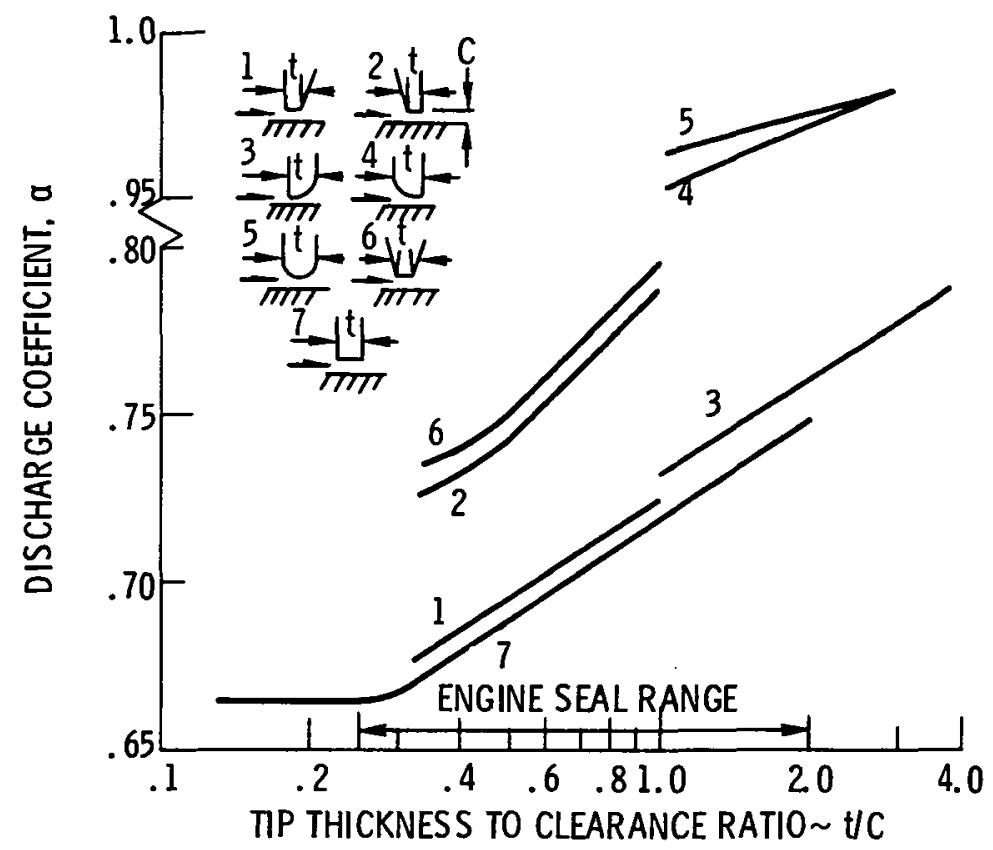

Figure 22. - Effect of knife-edge tooth shape on discharge coefficient (from ref. 1).

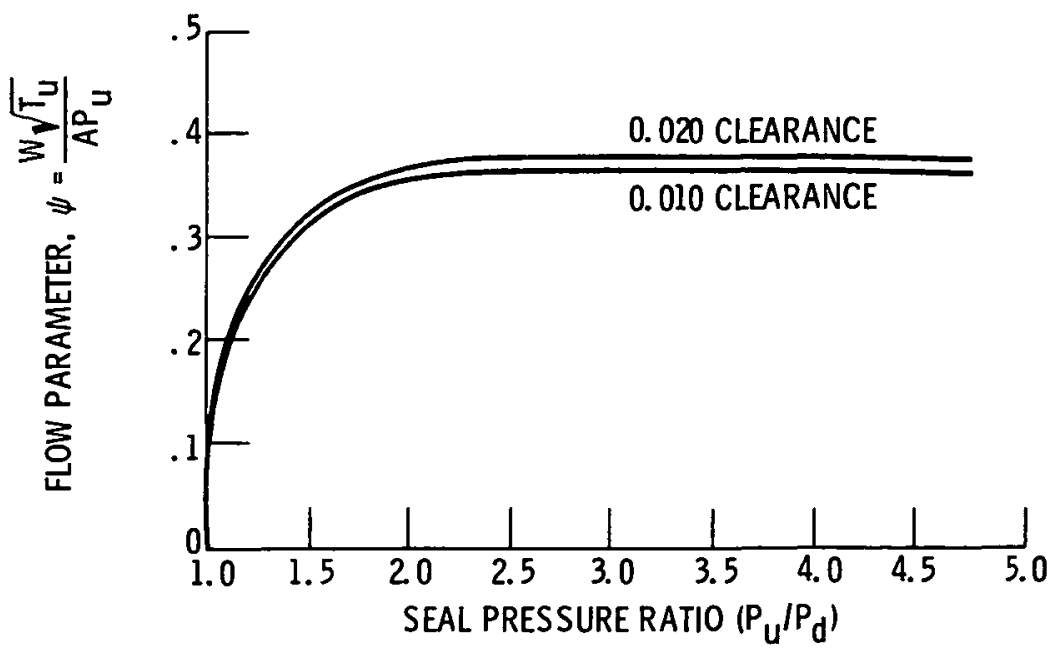

Figure 23. - Flow parameter as a function of pressure ratio; 4 tooth straight seal (ref. 23). 


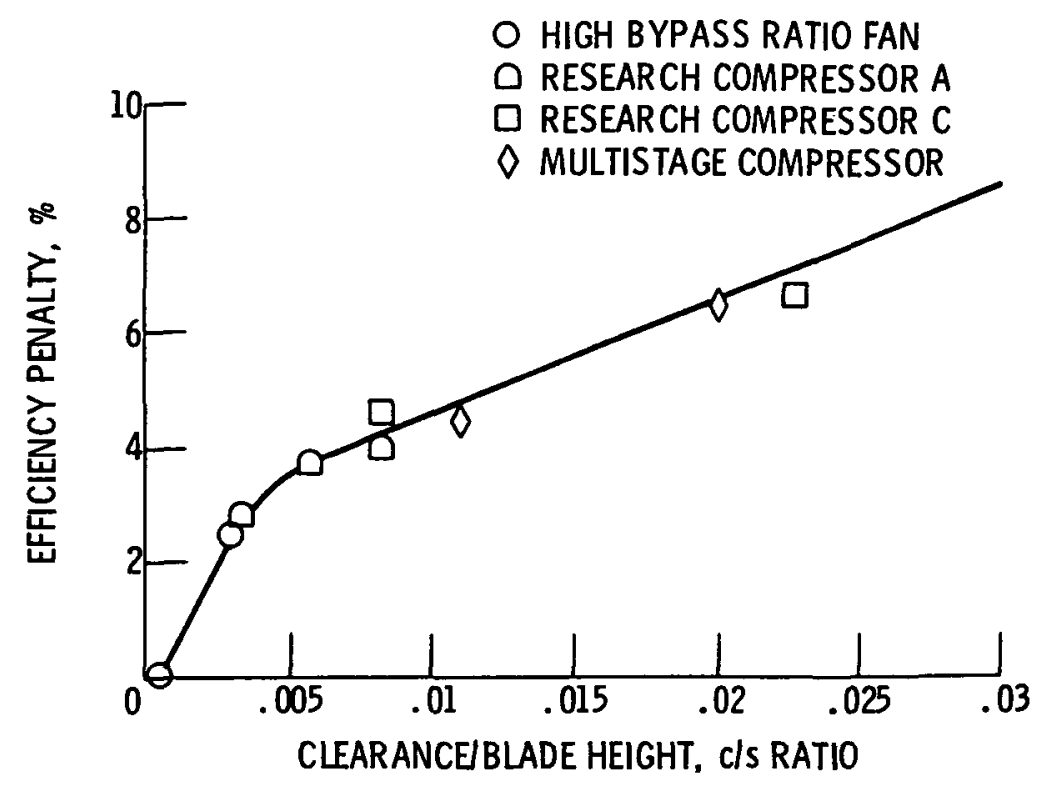

Figure 24. - Axial flow compressor polytropic efficiency penalty, effect of clearance to blade height ratio (ref. 1).

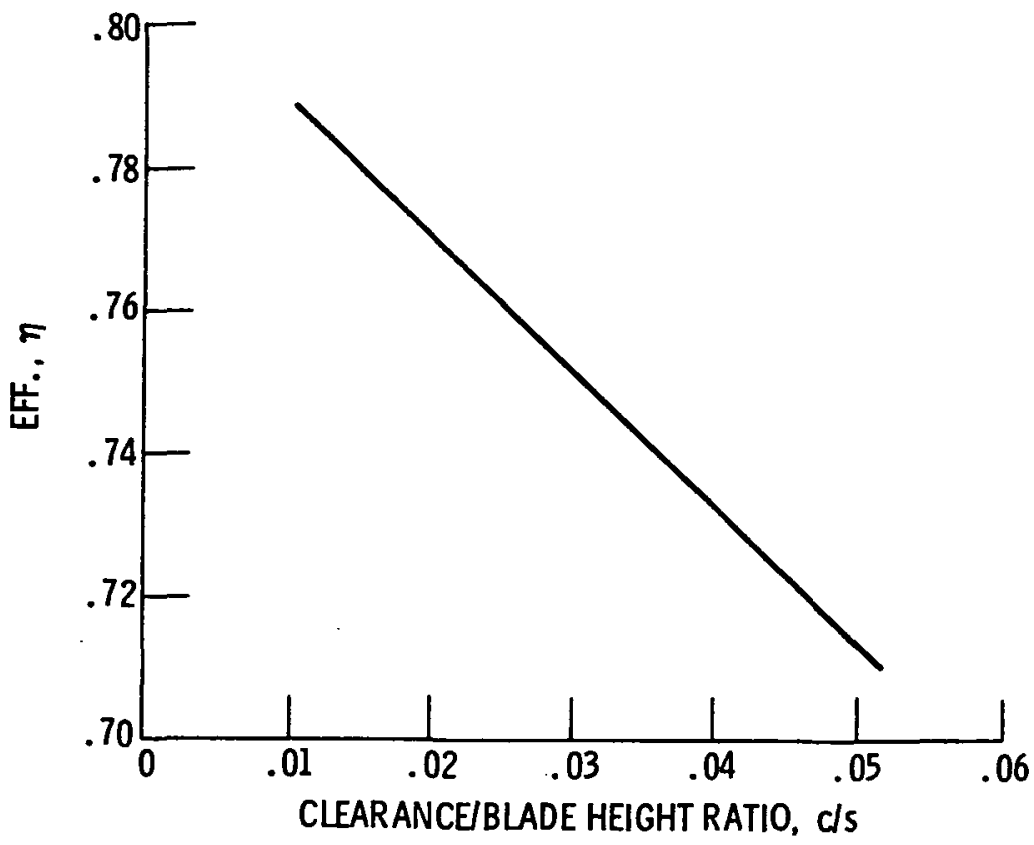

Figure 25. - Compressor efficiency as a function of $\mathrm{c} / \mathrm{s}$ ratio (ref. 25). 


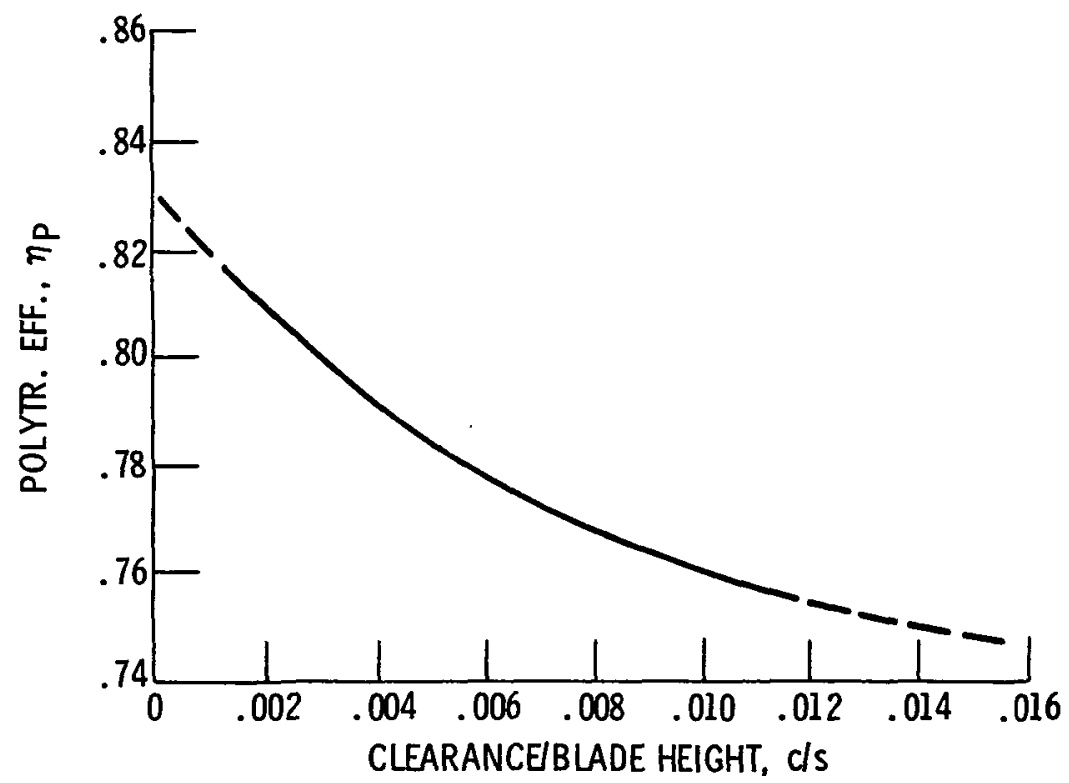

Figure 26. - Single stage fan blade tip clearance sensitivity, transition region (data from ref. 24).

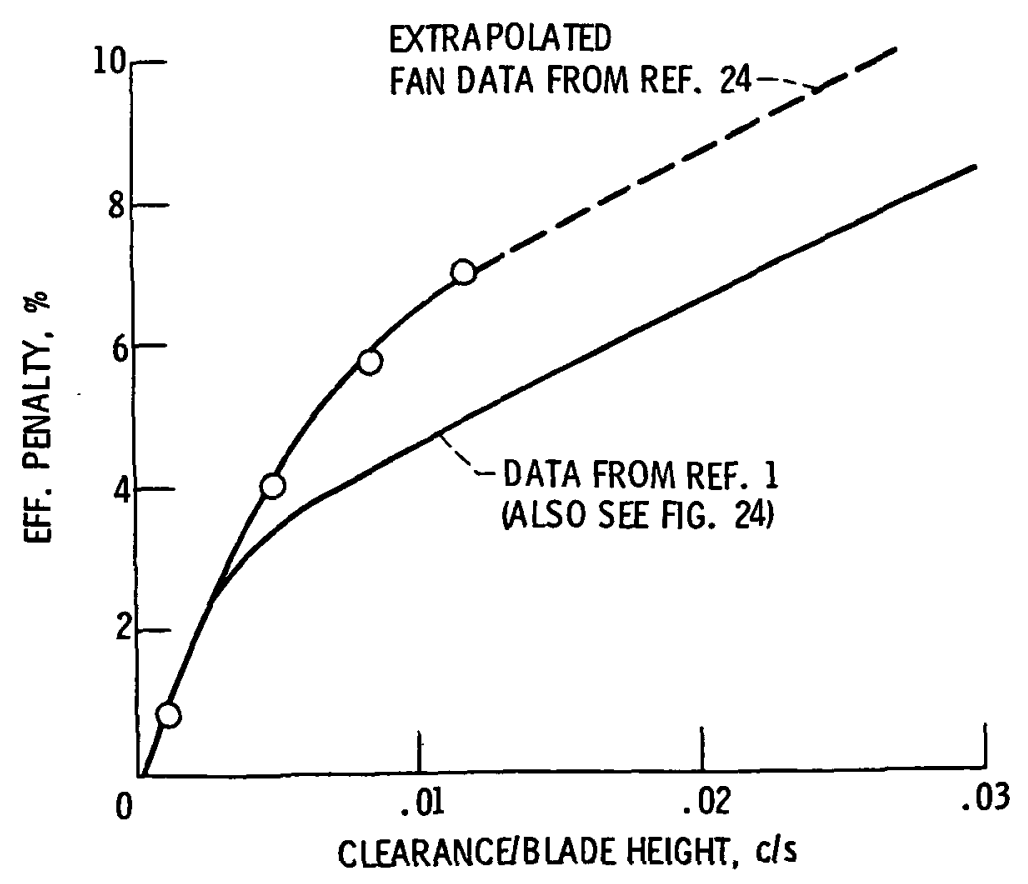

Figure 27. - Fan and compressor blade tip clearance sensitivity. 


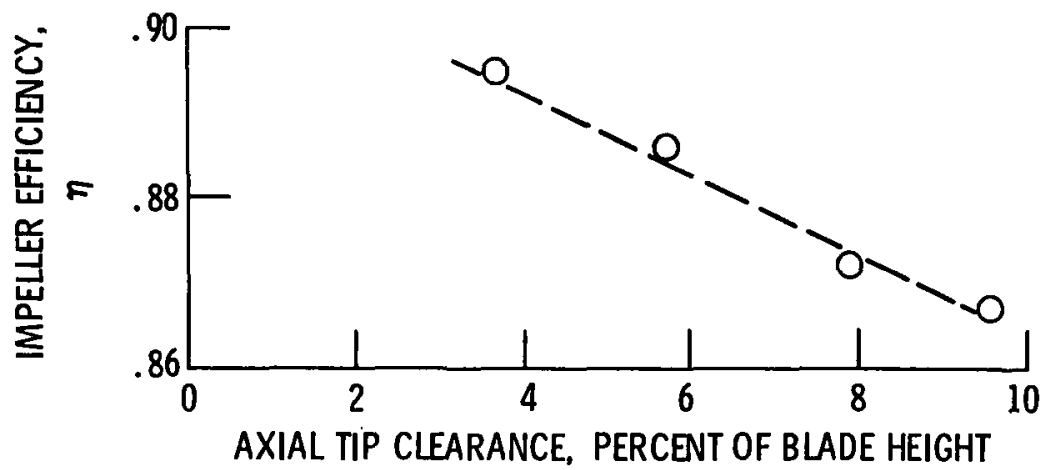

Figure 28. - Variation of impeller peak efficiency with axial tip clearance for vaneless diffuser test. Ratio of specific heat $\gamma=1.4$ (from ref. 30 ).

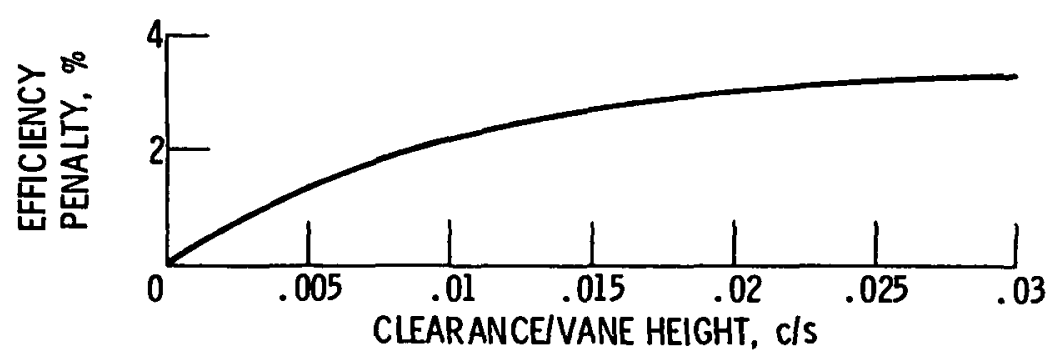

Figure 29. - Efficiency penalty as a function of clearance to vane height ratio, cantilevered vane and drum rotor (ref. 1).

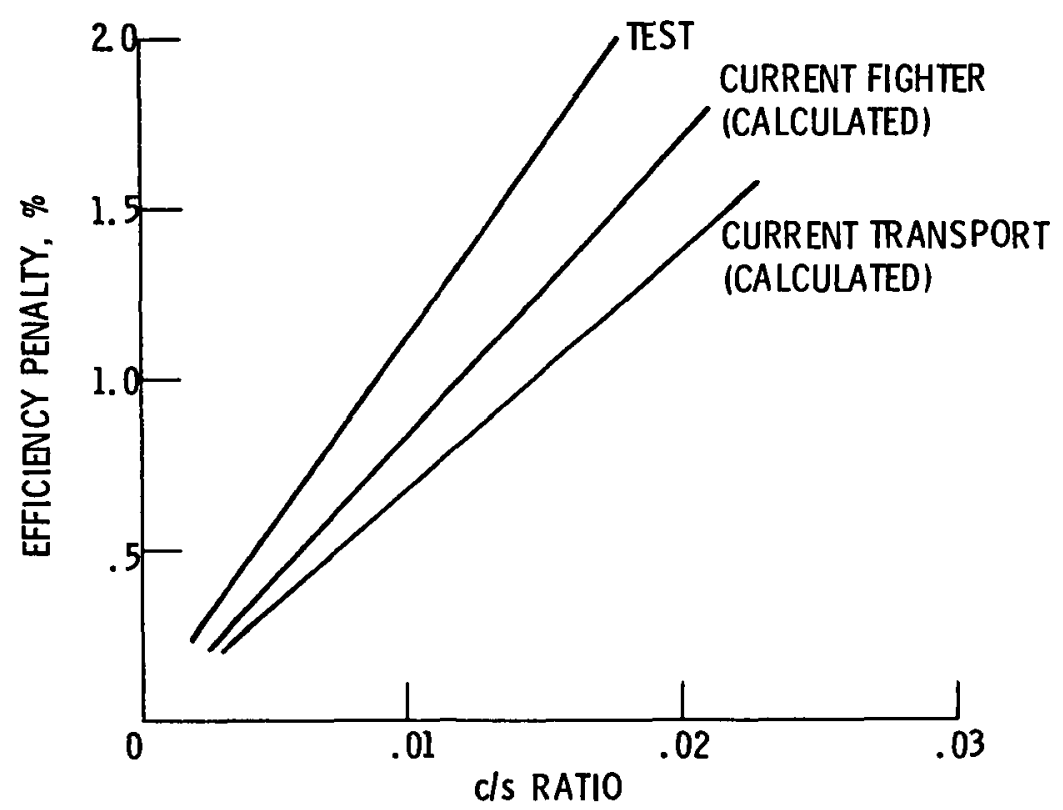

Figure 30. - Compressor efficiency penalty as a function of labyrinth clearance to vane height ratio (ref. 1). 

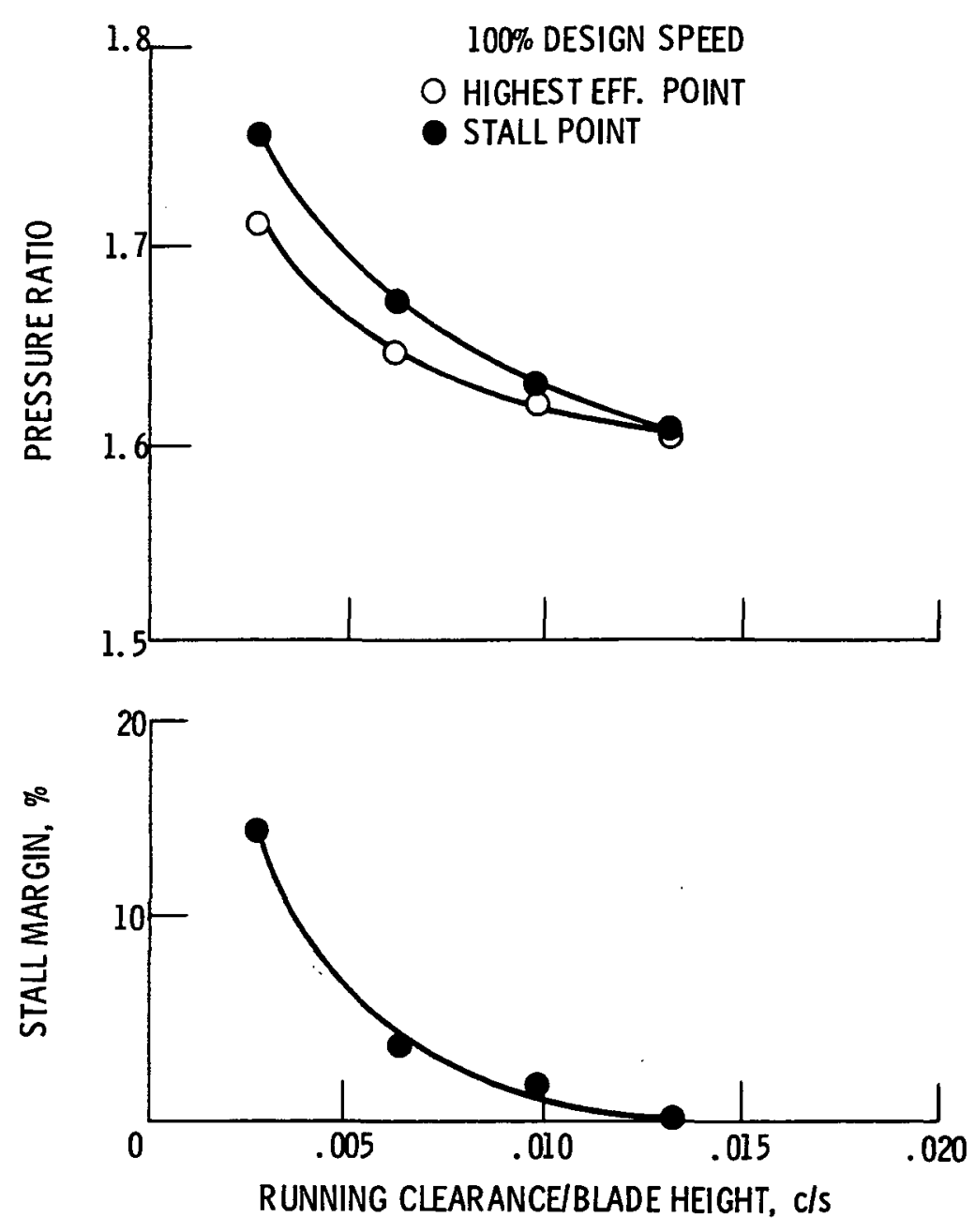

Figure 31. - Single stage fan, effect of blade clearance on pressure ratio and stall margin (ref. 24).

ORIGINAL PAG: ORIGINAL PUALITY

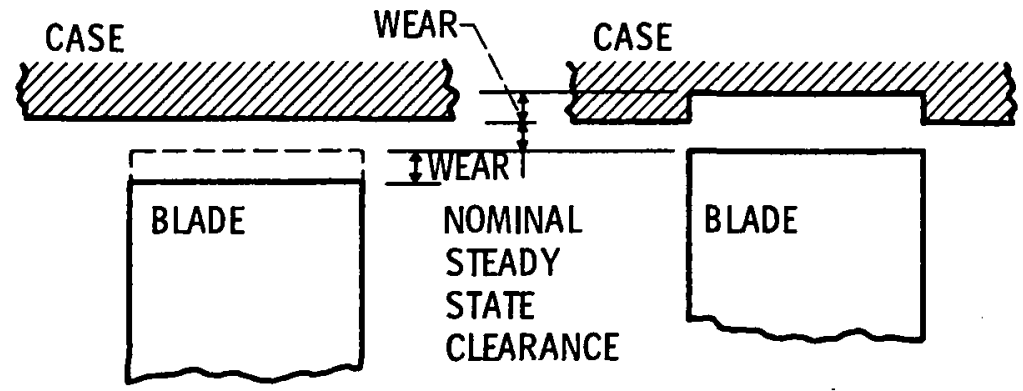

(a) TOTAL INTERFERENCE APPEARS AS BLADE WEAR.

(b) TOTAL INTERFERENCE APPEARS AS CASE WEAR.

Figure 32 - Illustration of two idealized extremes in wear caused by blade/case interference during transient operation. 


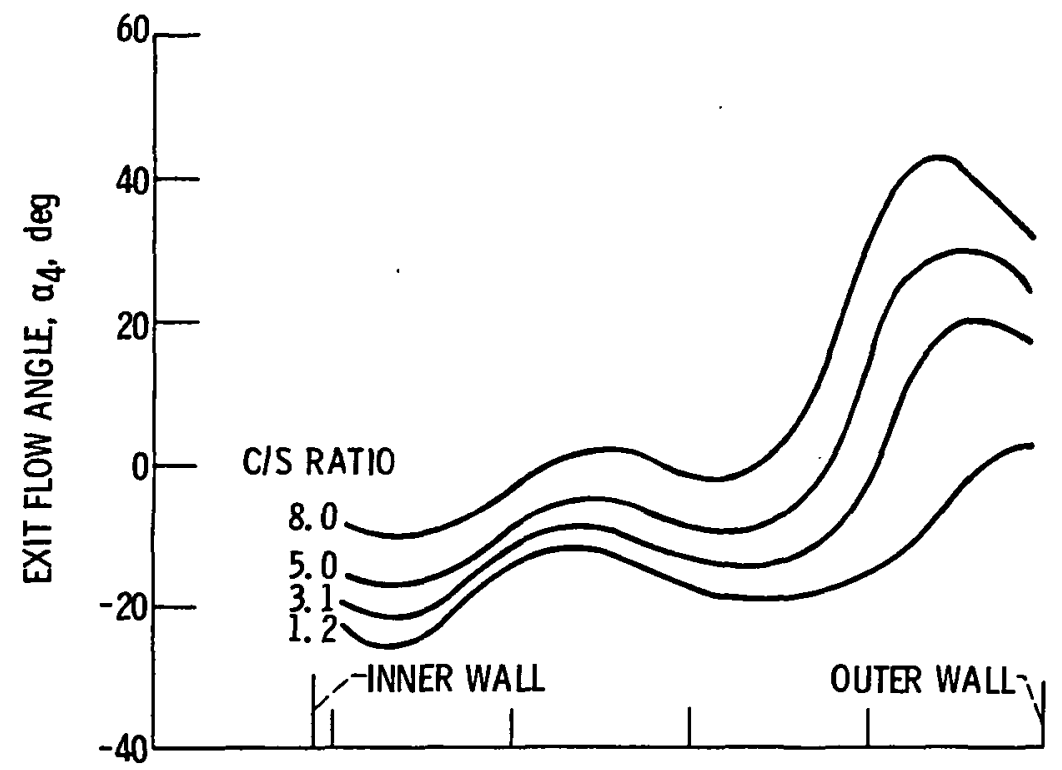

(a) VARIATION OF EXIT FLOW ANGLE WITH RADIUS RATIO.

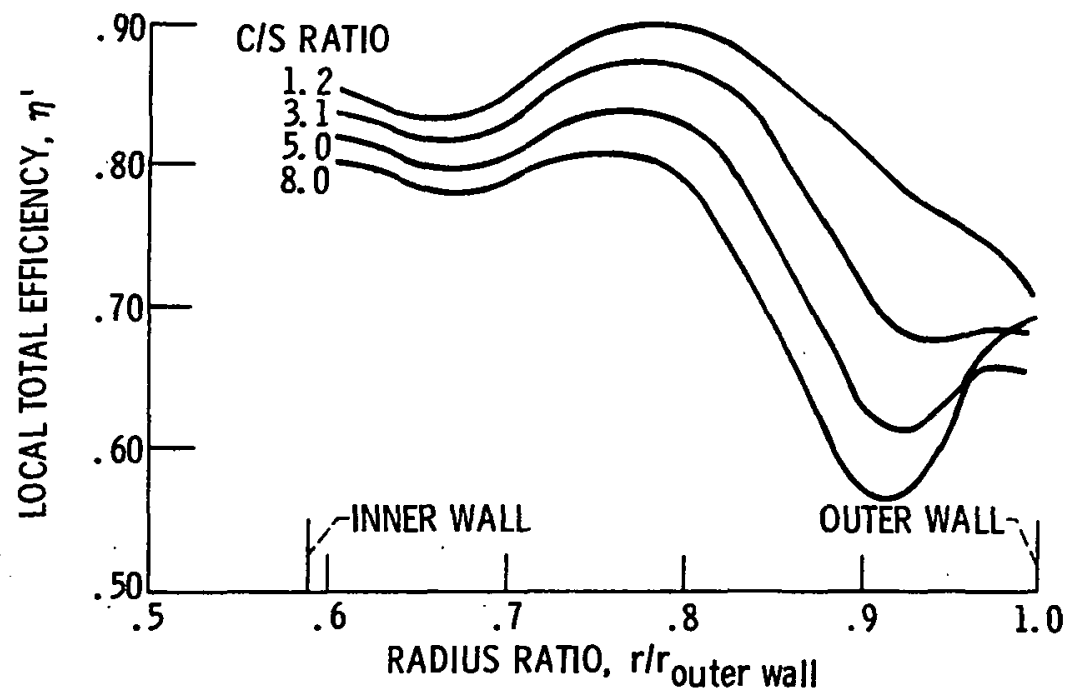

b) VARIATION OF TOTAL EFFICIENCY WITH RADIUS RATIO.

Figure 33. - Survey results at rotor exit at design equivalent speed and pressure ratio. (Ref. 31.) 
SOURCE TURBINE $R_{x} \frac{\left[1-\left(\eta / \eta_{0}\right)\right] \times 100}{\% \text { tip clearance }}$

$\left.\begin{array}{llll}\text { REF. } 32 & \text { IMPULSE } & 0 & 0.87 \\ \text { REF. 34 } & \text { REACTION } & .890 & 1.57\end{array}\right\}$ CASE-

REF. 32 IMPULSE 0

REF. 33 REACTION .805

REF. 34 REACTION .890

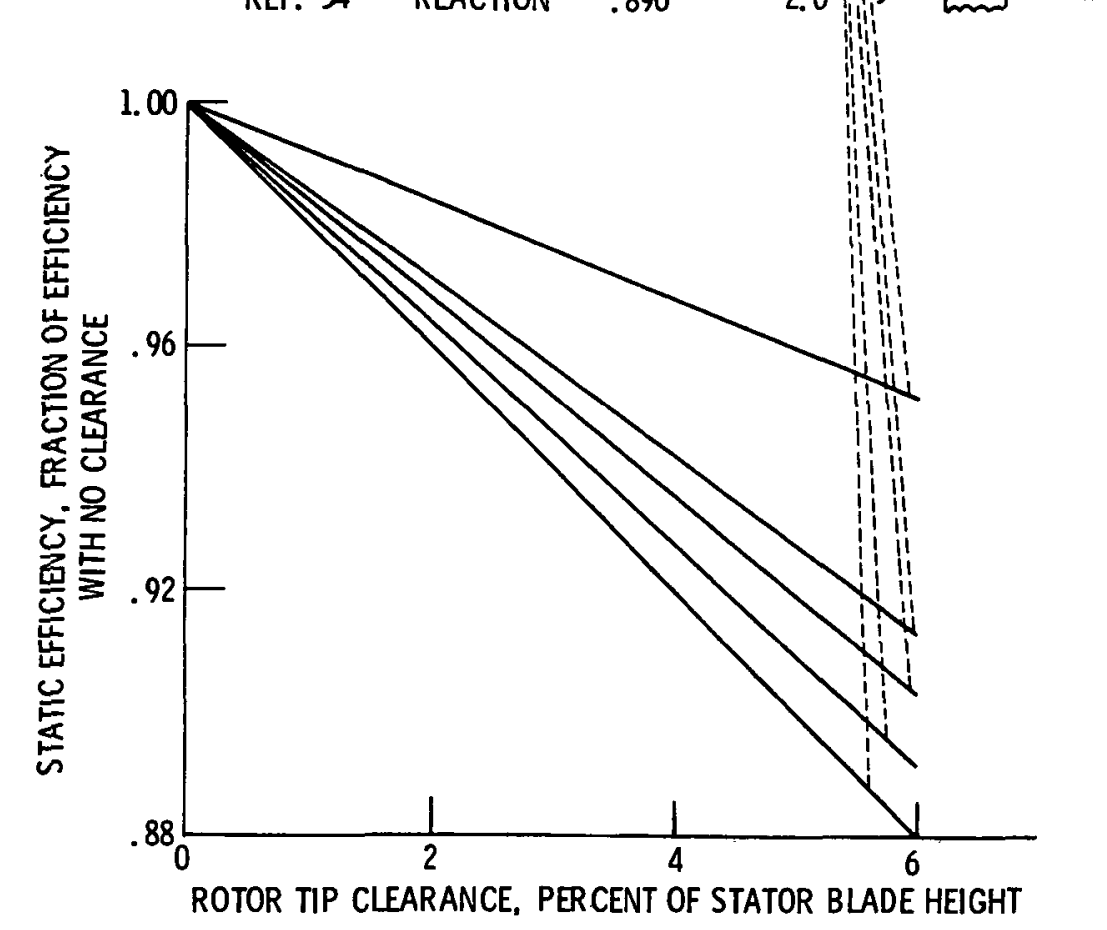

Figure 34. - Effect of rotor tip clearance on performance for various turbines (from ref. 34 ).

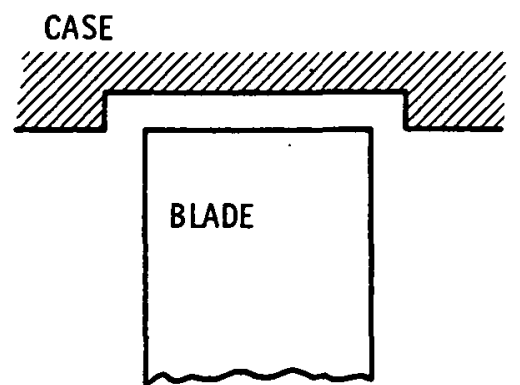

(a) BLADE TIP DIAMETER EQUAL TO CASE INSIDE DIAMETER (OPTIMUM GEOMEIRY).

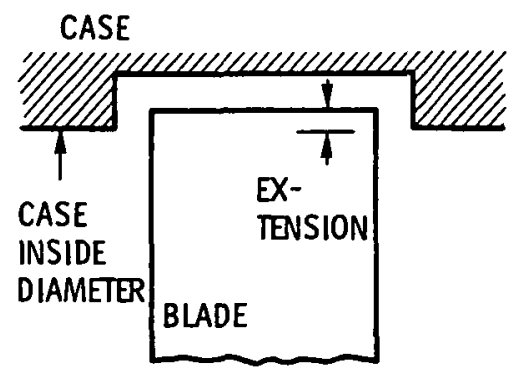

(D) BLADE TIP DIAMETER LARGER THAN CASE INSIDE DIAMETER.

Figure 35. - Recessed case configuration. 


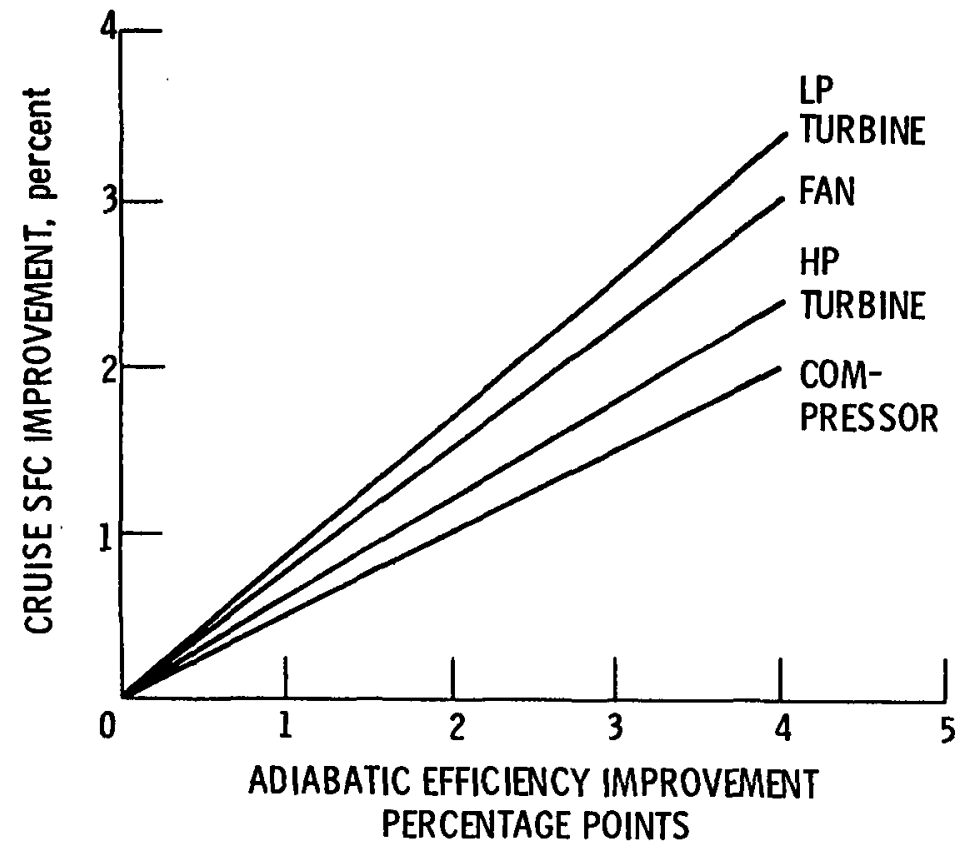

Figure 36. - Relation of SFC to component efficience (influence coefficients) in a high bypass engine (from ref. 151 .

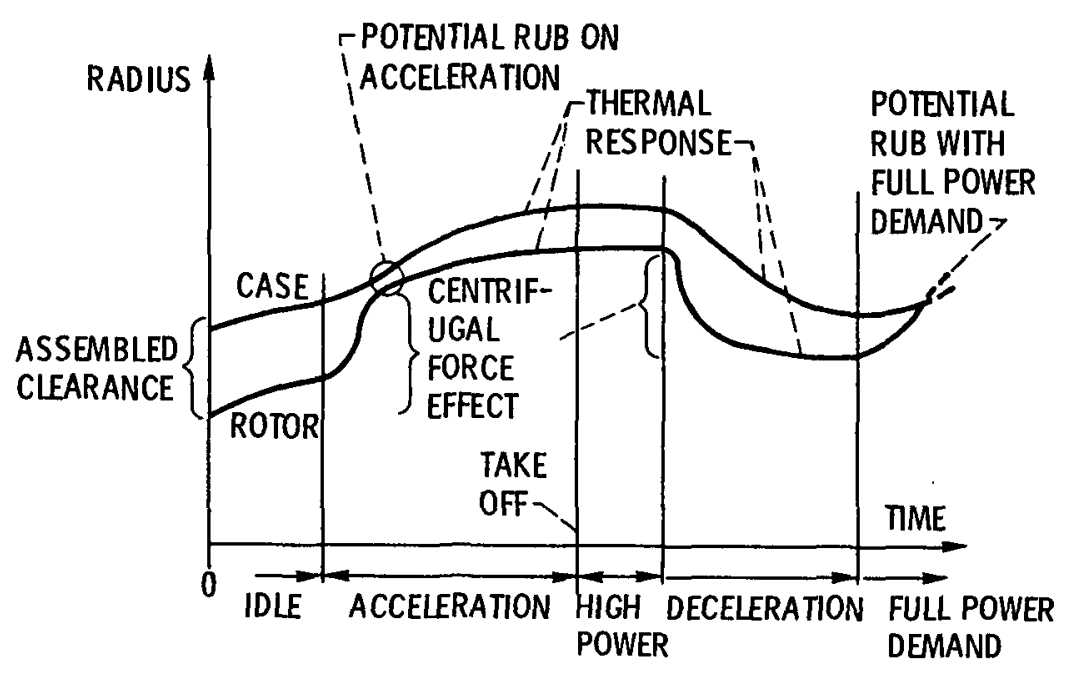

Figure 37. - Illustration of variation in case to rotor clearance during engine operation. 

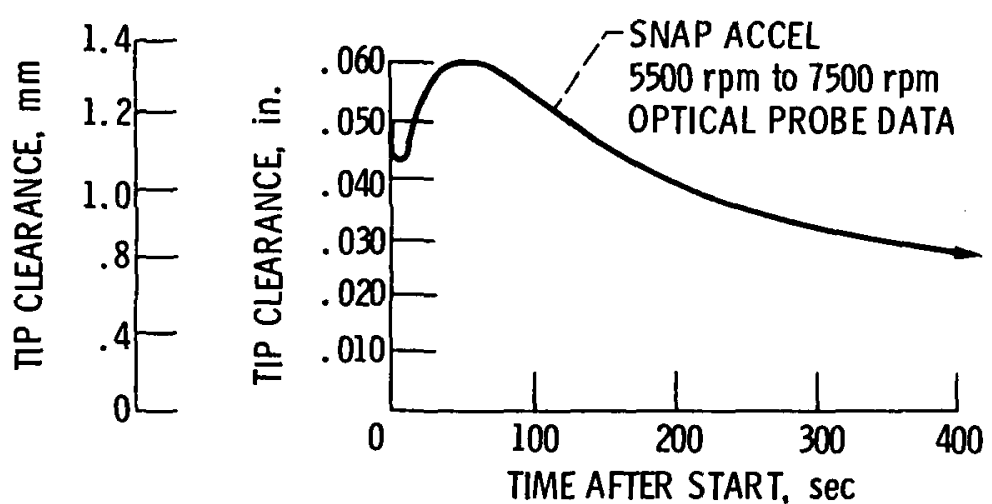

Figure 38. - Turbine blade tip clearance vs time (from ref. 37).

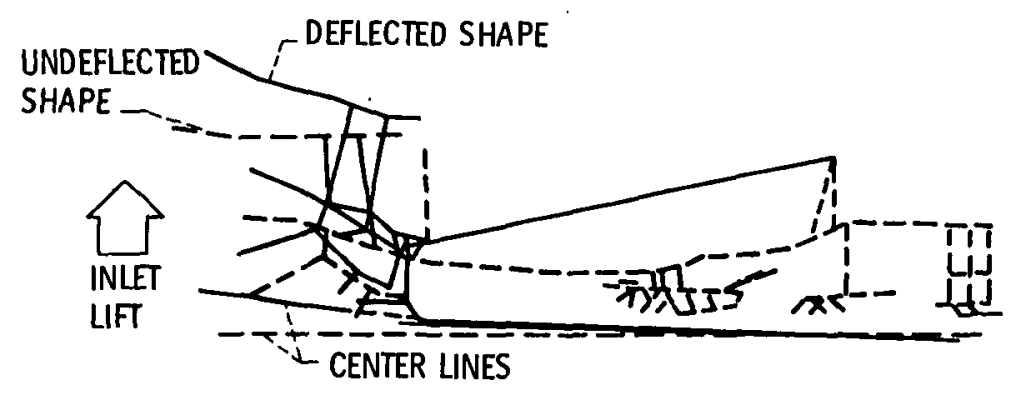

Figure 39. - Propulsion system static deflection due to inlet lift (from ref. 38).

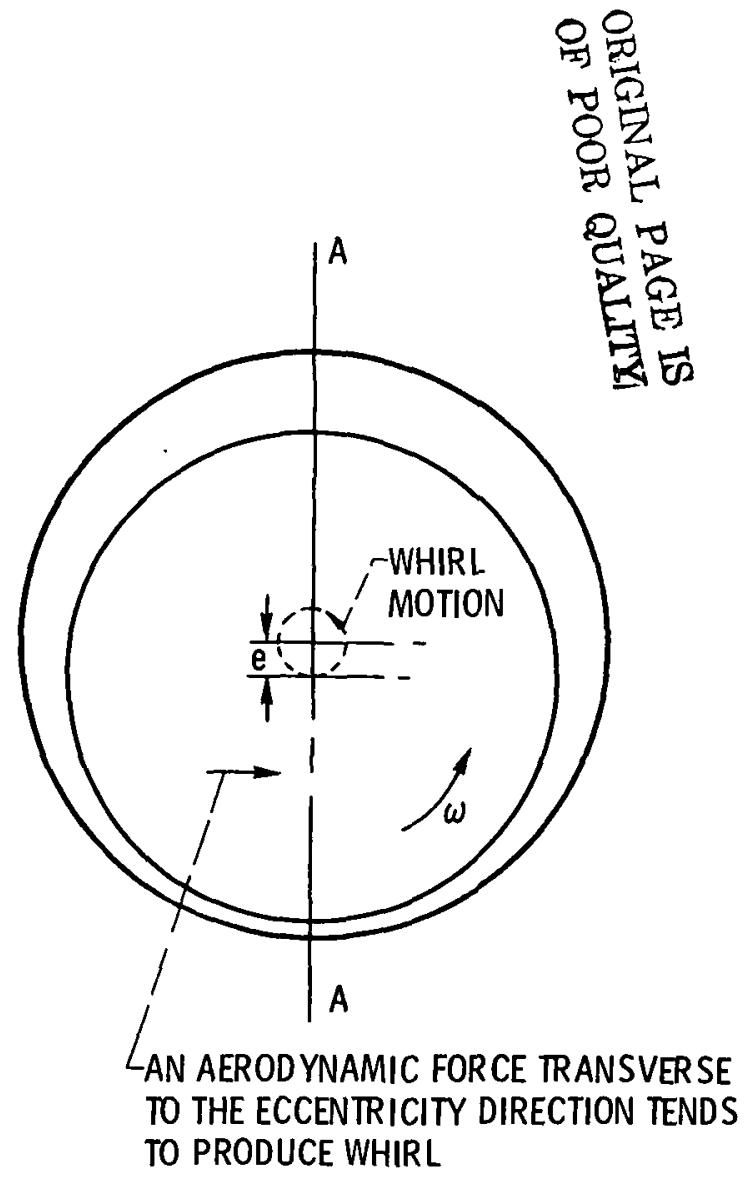

Figure 40. - Eccentric labyrinth seal and blade whirl model. 


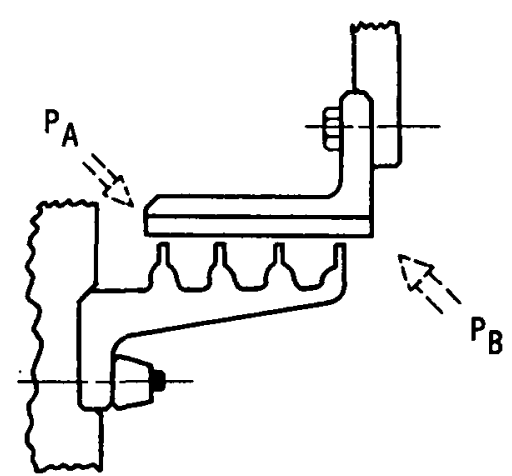

FOR $P_{A}>P_{B}$ ROTATING COMPONENTS SUPPORTED AT INLET HAVE FAILED, STATORS SUPPOR TED AT EXIT HAVE NOT FAILED

FOR $P_{B}>P_{A}$ ROTATING COMPONENTS SUPPORTED AT EXIT HAVE NOT FAILED, STATORS SUPPORTED AT INLET HAVE FAILED

Figure 4l. - Effect of seal component support.

POINT LIGHT SOURCE

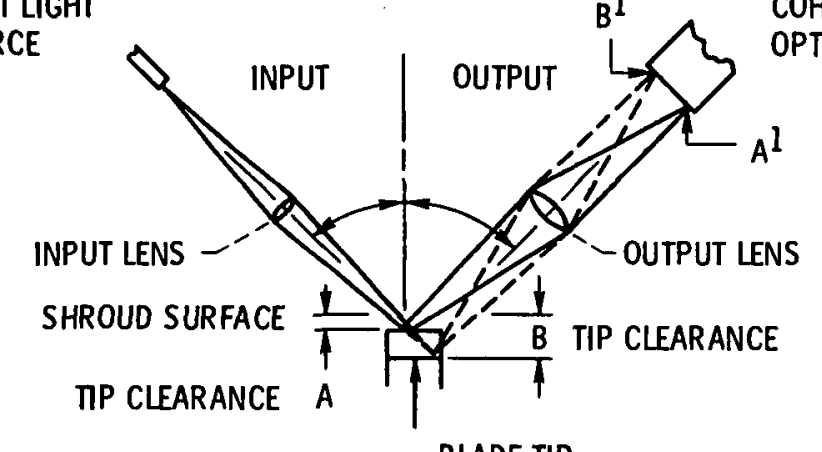

BLADE TIP

LIGHT REFLECTED FROM THE BLADE TIP IS FOCUSED THROUGH THE OUTPUT

LENS AND IS INCIDENT ON THE COHERENT FIBER OPTIC BUNDLE. AS THE BLADE TIP CLEARANCE INCREASES, THE OUTPUT BEAM MOVES FROM POSITION AI TO POSITION Bl.

Figure 42. - Laser optic clearance probe (ref. 43).
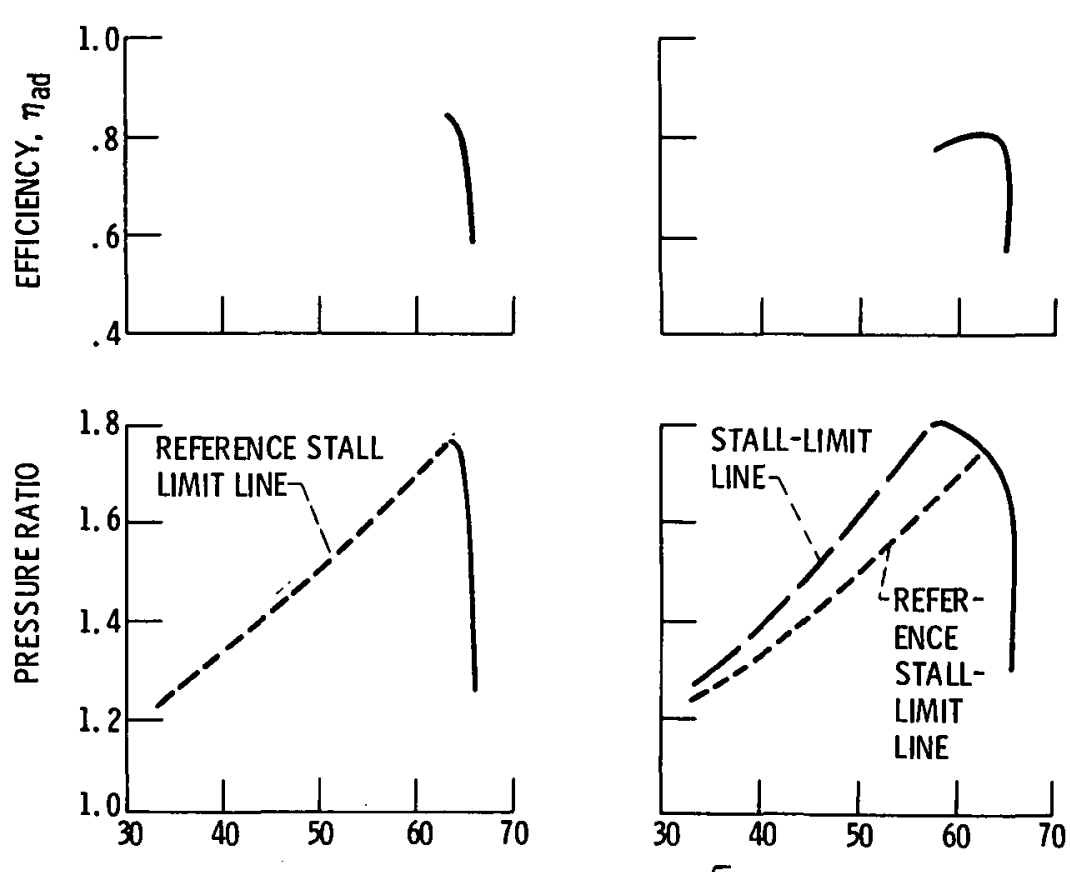

EQUIVALENT WEIGHT FLOW, $W \sqrt{\theta} / \delta, \mathrm{lbm} / \mathrm{sec}$

(a) SMOOTH CASING.

(b) HONEYCOMB CASING.

Figure 43. - Fan performance as a function of casing type, $100 \%$ design speed. 


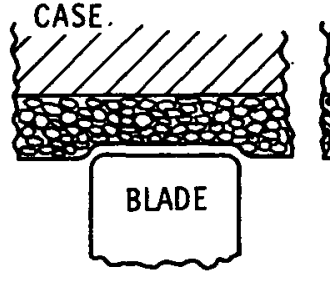

$\begin{array}{ll}\text { (a) ABRADABLE (SINTERED } & \text { b) COMPLIANT (POROUS }\end{array}$ OR SPRAYED POROUS MATERIALSI.

Figure 44. - Illustration of types of compressor rub materials for outer air sealing.

(a) STRIATED.

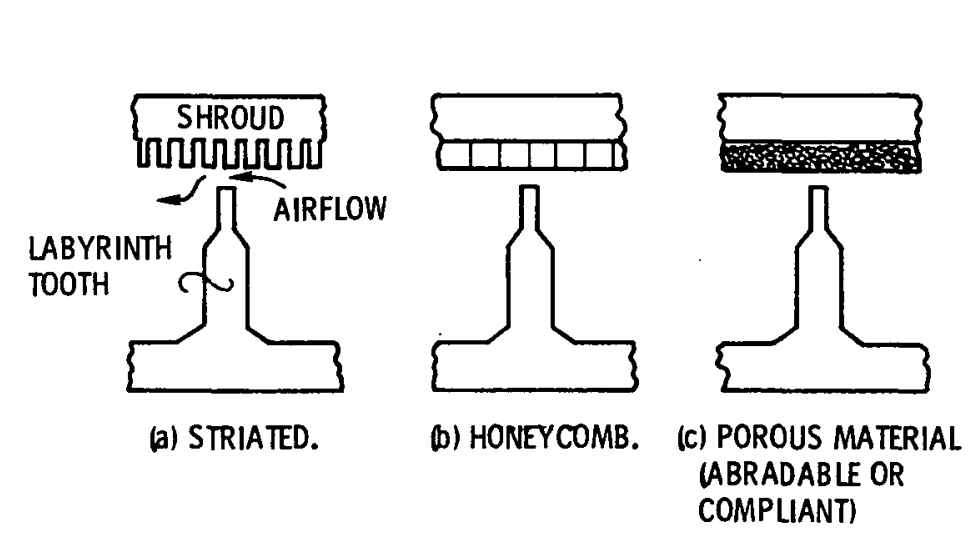

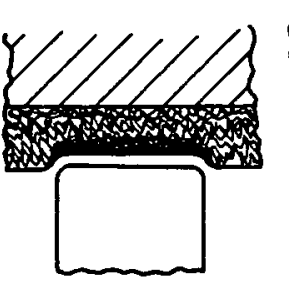
MATERIAL)

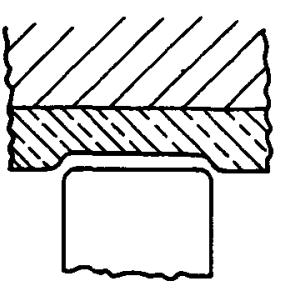

(c) LOW SHEAR STRENGTH (SPRAYED ALUMINUM)

Figure 45. - Inner shrouds for compressor interstage labyr inths. 


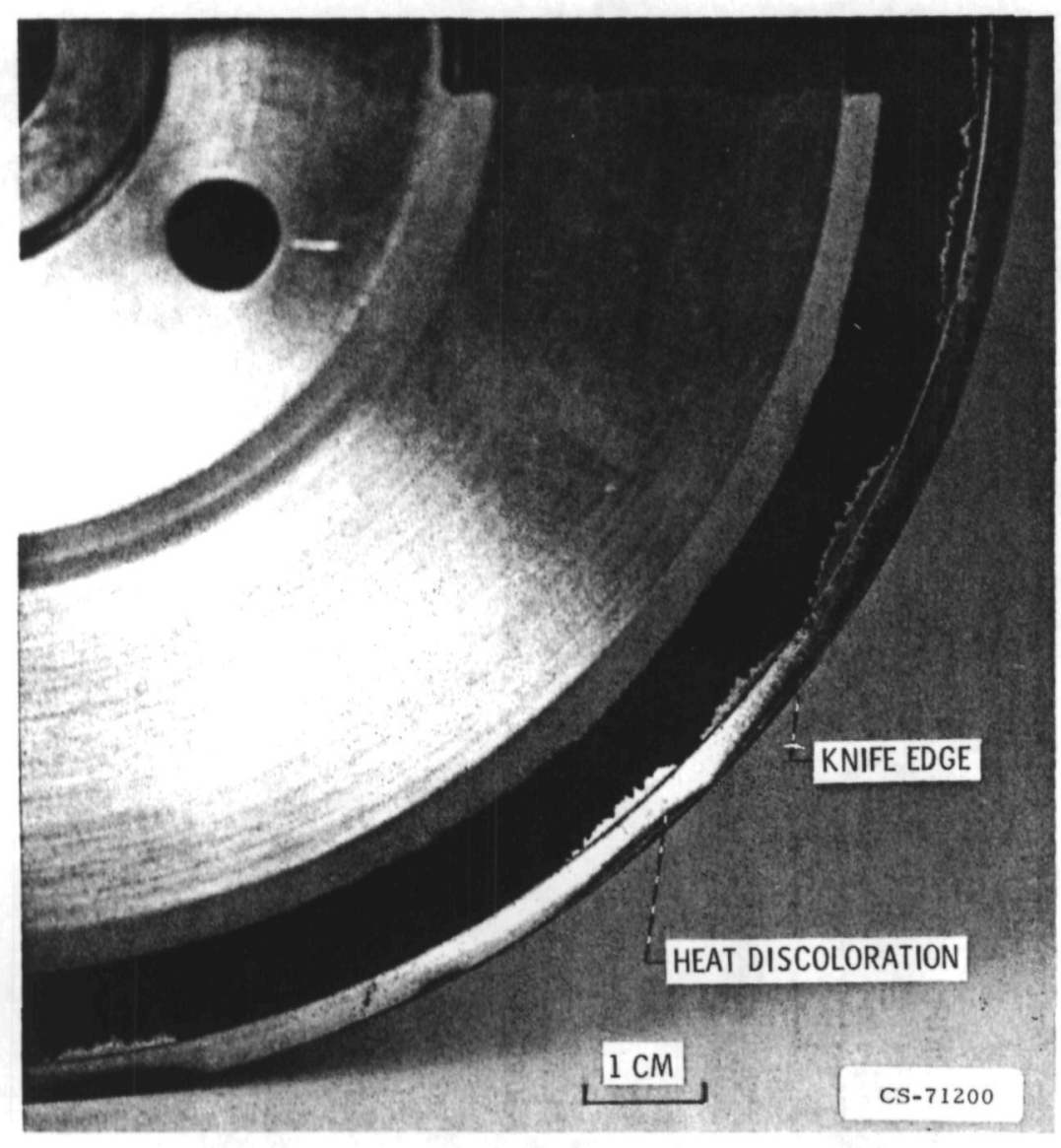

Figure 46. - Labyrinth disk and knife edge showing heat discoloration due to thermal bumps generated in rubbing contact against a shroud specimen. Rubbing speed, $183 \mathrm{~m} / \mathrm{s}(600 \mathrm{ft} / \mathrm{sec})$.

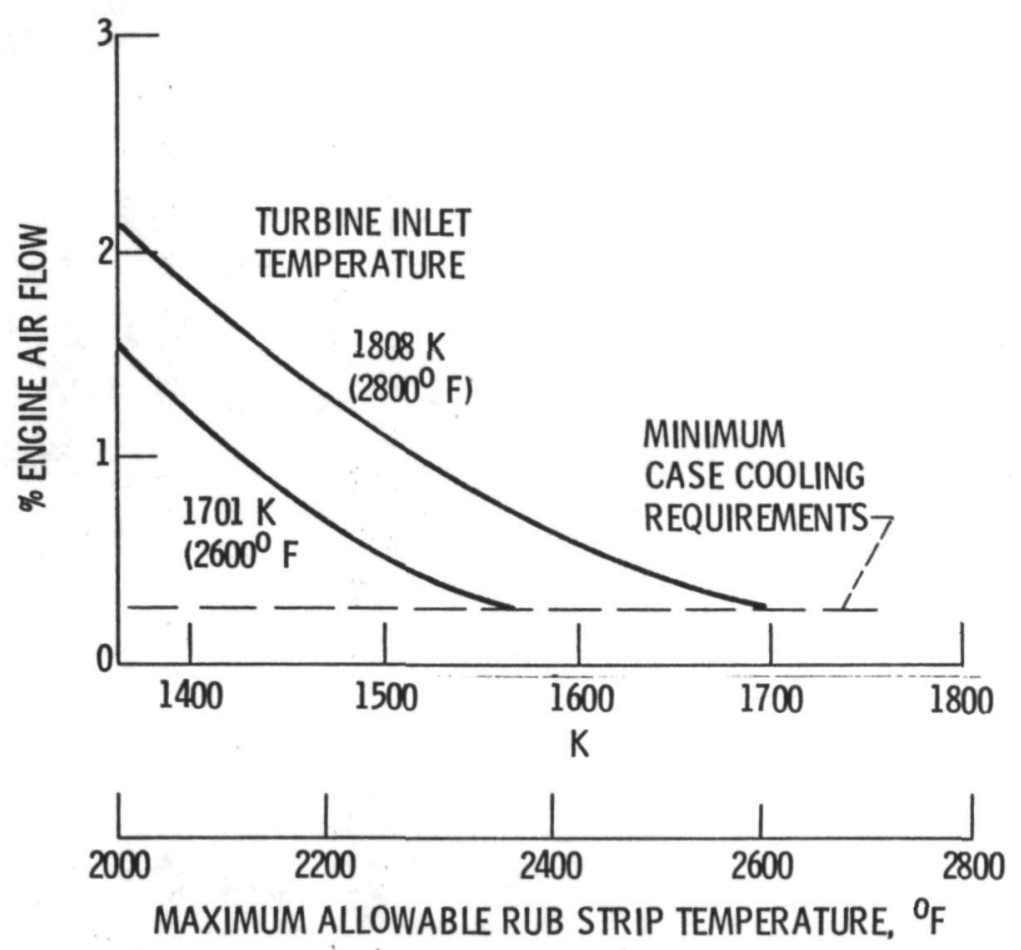

Figure 47. - Cooling air flow requirement for high pressure turbine outer air seal (data from ref. 52). 


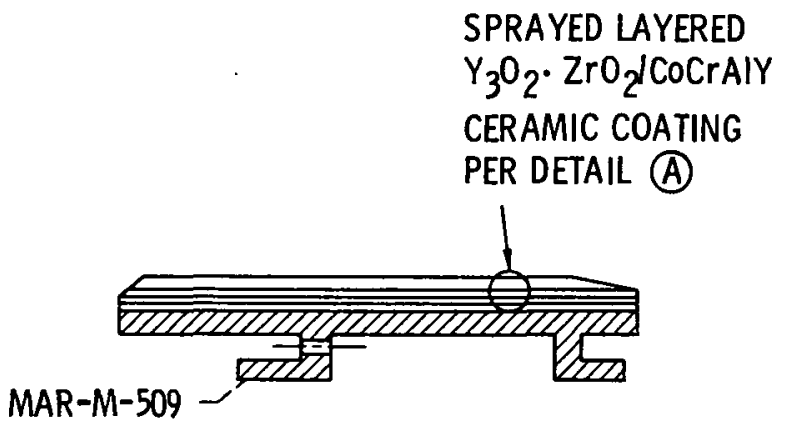

$\frac{4.216 \mathrm{~mm}(0.166 \mathrm{in.})}{3.607 \mathrm{~mm}(0.142 \mathrm{in.})}$

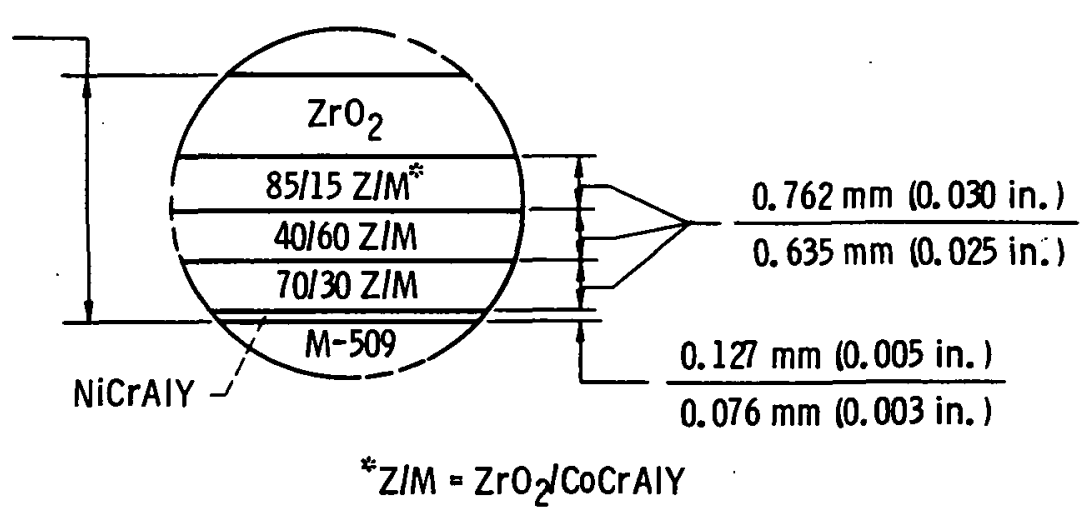

DETAIL A

Figure 48. - Typical engine seal segment (ref. 53). 